\title{
KMS conditions, standard real subspaces and reflection positivity on the circle group
}

\author{
Karl-Hermann Neeb, Gestur Ólafsson
}

\begin{abstract}
In the present paper we continue our investigations of the representation theoretic side of reflection positivity by studying positive definite functions $\psi$ on the additive group $(\mathbb{R},+)$ satisfying a suitably defined KMS condition. These functions take values in the space $\operatorname{Bil}(V)$ of bilinear forms on a real vector space $V$. As in quantum statistical mechanics, the KMS condition is defined in terms of an analytic continuation of $\psi$ to the strip $\{z \in \mathbb{C}: 0 \leq \operatorname{Im} z \leq \beta\}$ with a coupling condition $\psi(i \beta+t)=\overline{\psi(t)}$ on the boundary. Our first main result consists of a characterization of these functions in terms of modular objects $(\Delta, J)\left(J\right.$ an antilinear involution and $\Delta>0$ selfadjoint with $\left.J \Delta J=\Delta^{-1}\right)$ and an integral representation.

Our second main result is the existence of a $\operatorname{Bil}(V)$-valued positive definite function $f$ on the group $\mathbb{R}_{\tau}=\mathbb{R} \rtimes\left\{\operatorname{id}_{\mathbb{R}}, \tau\right\}$ with $\tau(t)=-t$ satisfying $f(t, \tau)=\psi(i t)$ for $t \in \mathbb{R}$. We thus obtain a $2 \beta$-periodic unitary one-parameter group on the GNS space $\mathcal{H}_{f}$ for which the one-parameter group on the GNS space $\mathcal{H}_{\psi}$ is obtained by Osterwalder-Schrader quantization.

Finally, we show that the building blocks of these representations arise from bundle-valued Sobolev spaces corresponding to the kernels $\left(\lambda^{2}-\frac{d^{2}}{d t^{2}}\right)^{-1}$ on the circle $\mathbb{R} / \beta \mathbb{Z}$ of length $\beta$.
\end{abstract}

\section{Introduction}

In this note we continue our investigations of the mathematical foundations of reflection positivity, a basic concept in constructive quantum field theory (GJ81, KL83, JÓl98, JÓl00, AFG86, JR07). Originally, reflection positivity, also called Osterwalder-Schrader positivity, arises as a requirement on the euclidean side to establish a duality between euclidean and relativistic quantum field theories ([OS73]). It is closely related to "Wick rotation" or "analytic continuation" in the time variable from the real to the imaginary axis.

The underlying fundamental concept is that of a reflection positive Hilbert space, introduced in [NÓ14a]. This is a triple $\left(\mathcal{E}, \mathcal{E}_{+}, \theta\right)$, where $\mathcal{E}$ is a Hilbert space, $\theta: \mathcal{E} \rightarrow \mathcal{E}$ is a unitary involution and $\mathcal{E}_{+}$is a closed subspace of $\mathcal{E}$ which is $\theta$-positive in the sense that $\langle\theta v, v\rangle \geq 0$ for $v \in \mathcal{E}_{+}$.

In NÓ14a] we introduced the concept of a reflection positive cyclic representation $(\pi, \mathcal{E}, v)$, where $\left(\mathcal{E}, \mathcal{E}_{+}, \theta\right)$ is a reflection positive Hilbert space and $v \in \mathcal{E}$ a $\theta$-fixed vector (or, more generally, a distribution vector). In the present paper we shall see that, to treat reflection positive representations of the circle group $G=\mathbb{T}$ corresponding to unitary representations of the dual group $G^{c} \cong \mathbb{R}$ arising from KMS states, resp., from their modular objects $(\Delta, J)[1$ we are forced to work in a more general framework, where the

\footnotetext{
${ }^{1}$ Recall that KMS stands for Kubo-Martin-Schwinger; see cite[§5.3.1]BR96 for more on KMS states and their interpretation in Quantum Statistical Mechanics as thermal equilibrium states.
} 
representations are generated by the image of an $\mathbb{R}$-linear map $j: V \rightarrow \mathcal{E}$ from a real vector space $V$ into the representation space $\mathcal{E}$ and where $j(V)$ does not consist of $\theta$-fixed vectors.

To explain the corresponding concept of a reflection positive representation, we start with a symmetric Lie group, i.e., a pair $(G, \tau)$, where $\tau \in \operatorname{Aut}(G)$ is an involution. Then we form the extended group $G_{\tau}:=G \rtimes\{\mathbf{1}, \tau\}$. Let $(U, \mathcal{E})$ be a unitary representation of $G_{\tau}$ and let $j: V \rightarrow \mathcal{E}$ be a linear map from the real vector space $V$ to $\mathcal{E}$. Then $(U, \mathcal{E}, j, V)$ is called reflection positive with respect to a subset $G_{+} \subseteq G$ if the closed subspace $\mathcal{E}_{+}$generated by $U_{G_{+}}^{-1} j(V)$ defines a reflection positive Hilbert space $\left(\mathcal{E}, \mathcal{E}_{+}, U_{\tau}\right)$. Generalizing the well-known Gelfand-Naimark-Segal (GNS) construction leads to an encoding of representations generated by $j(V)$ in terms of form-valued positive definite functions $\psi(g)(v, w):=$ $\left\langle j(v), U_{g} j(w)\right\rangle($ NÓ15] $)$.

This paper continues the investigations started in NÓ15], where we studied reflection positive representations of the circle group and their connections to KMS states, which was largely motivated by the work of Klein and Landau in [KL81] (see also [CMV01]). A long term goal of this project is to combine our representation theoretic approach to reflection positivity with KMS states of operator algebras and Borchers triples corresponding to modular inclusions (BLS11], Bo92, [Lo08, Sch99]).

A crucial step in this direction is the concept of a positive definite function satisfying a KMS condition that can be formulated as follows: First, let $V$ be a real vector space and $\operatorname{Bil}(V)$ be the space of real bilinear maps $V \times V \rightarrow \mathbb{C}$. A function $\psi: \mathbb{R} \rightarrow \operatorname{Bil}(V)$ is said to be positive definite if the kernel $\psi(t-s)(v, w)$ on $\mathbb{R} \times V$ is positive definite. For $\beta>0$, we consider the open strip $\mathcal{S}_{\beta}:=\{z \in \mathbb{C}: 0<\operatorname{Im} z<\beta\}$. We say that a positive definite function $\psi: \mathbb{R} \rightarrow \operatorname{Bil}(V)$ satisfies the $K M S$ condition for $\beta>0$ if $\psi$ extends to a function $\overline{\mathcal{S}_{\beta}} \rightarrow \operatorname{Bil}(V)$ which is pointwise continuous and pointwise holomorphic on the interior $\mathcal{S}_{\beta}$, and satisfies

$$
\psi(i \beta+t)=\overline{\psi(t)} \quad \text { for } \quad t \in \mathbb{R} .
$$

The central idea in the classification of positive definite functions satisfying a KMS condition is to relate them to standard real subspaces of a (complex) Hilbert space; these are closed real subspaces $V \subseteq \mathcal{H}$ for which $V \cap i V=\{0\}$ and $V+i V$ is dense (cf. Definition 2.4). Any such subspace determines a pair $(\Delta, J)$ of modular objects, where $\Delta$ is a positive selfadjoint operator and $J$ an antilinear involution satisfying $J \Delta J=\Delta^{-1}$. The connection is established by $V=\operatorname{Fix}\left(J \Delta^{1 / 2}\right)=\left\{v \in \mathcal{D}\left(\Delta^{1 / 2}\right): J \Delta^{1 / 2} v=v\right\}$ Our first main result is the following characterization of the KMS condition in terms of standard real subspaces. Here we write $\operatorname{Bil}^{+}(V) \subseteq \operatorname{Bil}(V)$ for the convex cone of all those bilinear forms $f$ for which the sesquilinear extension to $V_{\mathbb{C}} \times V_{\mathbb{C}}$ is positive semidefinite.

Theorem 1.1. (Characterization of the KMS condition) Let $V$ be a real vector space and let $\psi: \mathbb{R} \rightarrow$ $\operatorname{Bil}(V)$ be a pointwise continuous positive definite function. Then the following are equivalent:

(i) $\psi$ satisfies the $K M S$ condition for $\beta>0$.

(ii) There exists a standard real subspace $V_{1}$ in a Hilbert space $\mathcal{H}$ and a linear map $j: V \rightarrow V_{1}$ such that

$$
\psi(t)(v, w)=\left\langle j(v), \Delta^{-i t / \beta} j(w)\right\rangle \quad \text { for } \quad t \in \mathbb{R}, v, w \in V .
$$

(iii) There exists a $\mathrm{Bil}^{+}(V)$-valued regular Borel measure $\mu$ on $\mathbb{R}$ satisfying

$$
\psi(t)=\int_{\mathbb{R}} e^{i t \lambda} d \mu(\lambda), \quad \text { where } \quad d \mu(-\lambda)=e^{-\beta \lambda} d \bar{\mu}(\lambda) .
$$

If these conditions are satisfied, then the function $\psi: \overline{\mathcal{S}_{\beta}} \rightarrow \operatorname{Bil}(V)$ is pointwise bounded. 
The equivalence of (i) and (ii) in Theorem 1.1 describes the tight connection between the KMS condition and the modular objects associated to a standard real subspace. Part (iii) provides an integral representation that can be viewed as a classification result.

For a function $\psi$ satisfying the $\beta$-KMS condition, analytic continuation leads to the operator-valued function

$$
\varphi:[0, \beta] \rightarrow B\left(V_{\mathbb{C}}\right), \quad\langle v, \varphi(t) w\rangle=\psi(i t)(v, w) .
$$

This function satisfies $\varphi(\beta)=\overline{\varphi(0)}$, hence extends uniquely to a (weak operator) continuous function $\varphi: \mathbb{R} \rightarrow B\left(V_{\mathbb{C}}\right)$ satisfying

$$
\varphi(t+\beta)=\overline{\varphi(t)} \quad \text { for } \quad t \in \mathbb{R} .
$$

Recall the group $\mathbb{R}_{\tau}:=\mathbb{R} \rtimes\{1, \tau\}$ with $\tau(t)=-t$. In Theorem 4.5 we show that there exists a positive definite function

$$
f: \mathbb{R}_{\tau} \rightarrow \operatorname{Bil}(V) \quad \text { satisfying } \quad f(t, \tau)=\widehat{\varphi}(t) .
$$

The function $f$ is $2 \beta$-periodic, hence factors through a function on $\mathbb{T}_{2 \beta, \tau}:=\mathbb{R}_{\tau} / \mathbb{Z} 2 \beta \cong \mathrm{O}_{2}(\mathbb{R})$. This leads to a natural "euclidean" counterpart of the unitary one-parameter group $U_{t}=\Delta^{-i t / \beta}$ associated to the KMS positive definite function $\psi$. To understand the structure of the so arising positive definite functions and the corresponding unitary representations of $\mathbb{T}_{2 \beta, \tau}$, we write $f=f_{+}+f_{-}$with $f_{+}\left(\beta+t, \tau^{\varepsilon}\right)=f_{+}\left(t, \tau^{\varepsilon}\right)$ (the bosonic part) and $f_{-}\left(\beta+t, \tau^{\varepsilon}\right)=-f_{-}\left(t, \tau^{\varepsilon}\right)$ (the fermionic part). Then $f_{ \pm}$are both positive definite and combine to a matrix valued positive definite function

$$
f^{\sharp}:=\left(\begin{array}{cc}
f_{+} & 0 \\
0 & f_{-}
\end{array}\right): \mathbb{R}_{\tau} \rightarrow M_{2}\left(B\left(V_{\mathbb{C}}\right)\right) \cong B\left(V_{\mathbb{C}}^{2}\right)
$$

(Lemma 4.12). Neglecting an additive summand which is constant, we can now defined a unitary representation of the subgroup $P:=(\mathbb{Z} \beta)_{\tau}$ on $V_{\mathbb{C}}^{2}$ by

$$
\rho(\beta, \mathbf{1}):=\left(\begin{array}{cc}
\mathbf{1} & 0 \\
0 & -\mathbf{1}
\end{array}\right) \quad \text { and } \quad \rho(0, \tau):=\left(\begin{array}{cc}
\mathbf{1} & 0 \\
0 & i I
\end{array}\right),
$$

where $I$ is a complex structure on $V$. Then we have the relation

$$
f^{\sharp}(h g)=\rho(h) f^{\sharp}(g) \quad \text { for } \quad h \in P, g \in \mathbb{R}_{\tau}
$$

which determines in particular how $f^{\sharp}$ is obtained from the function $\varphi$ from above.

For the special case where the real representation corresponding to $\psi$ is isotypic, resp., the associated modular operator $\Delta$ is a multiple of the identity, the GNS representation $\left(U^{f^{\sharp}}, \mathcal{H}_{f^{\sharp}}\right)$ can be realized on the Hilbert space completion of

$$
\Gamma_{\rho}:=\left\{s \in C^{\infty}\left(\mathbb{R}_{\tau}, V_{\mathbb{C}}^{2}\right):\left(\forall g \in \mathbb{R}_{\tau}, h \in P\right) s(h g)=\rho(h) s(g)\right\}
$$

with respect to the scalar product

$$
\left\langle s_{1}, s_{2}\right\rangle:=\frac{1}{2 \beta} \int_{0}^{2 \beta}\left\langle s_{1}(t, \mathbf{1}),\left(\left(\lambda^{2}-\Delta\right)^{-1} s_{2}\right)(t, \mathbf{1})\right\rangle d t, \quad \text { where } \quad \Delta=\frac{d^{2}}{d t^{2}} .
$$

On this space $\mathbb{R}_{\tau}$ acts by right translation. This provides a natural "euclidean realization" of our representation on the Riemannian manifold $\mathbb{T}_{\beta} \cong \mathbb{S}^{1}$ in the spirit of [AFG86, Di04, JR07. 
We conclude this paper with a short Section [5, in which we prove a version of Theorem 1.1 for $\beta=\infty$ which connects naturally to our previous work on dialations of semigroups of contractions in $[\mathrm{NÓ} 14 \mathrm{~b}$. In two appendices we provide some background material. Appendix @ recalls some facts on positive definite kernels and discusses in particular the connection between complex and real-valued kernels. Appendix B discusses standard real subspaces in terms of skew-symmetric contractions on real Hilbert spaces. This perspective was crucial for the present paper, and we expect it to be useful in other contexts as well.

In a subsequent paper [NÓ16] we extend the results obtained here for the group $G=\mathrm{O}_{2}(\mathbb{R})=\mathrm{SO}_{2}(\mathbb{R})_{\tau}$ to more general groups such as $\mathrm{O}_{n+1}(\mathbb{R})$ (where reflection positivity refers to the sphere $\mathbb{S}^{n}$ ) and $\mathrm{O}_{1, n}(\mathbb{R}$ ) (where reflection positivity refers to the $n$-dimensional hyperbolic space $\mathbb{H}^{n}$ ). Eventually, we would like to see how our representation theoretic analysis can be blended with the existing work on relativistic KMS conditions [BB94, GJ06] and in particular with [BJM13, BJM15].

The close connection between modular objects $(\Delta, J)$ and standard real subspaces was first explored by Rieffel and van Daele in RvD77. They also define a notion of a KMS condition for a unitary oneparameter group $\left(U_{t}\right)_{t \in \mathbb{R}}$ on a complex Hilbert space $\mathcal{H}$ with a real subspace $V \subseteq \mathcal{H}$. In our terms, their condition means that the function $\psi: \mathbb{R} \rightarrow \operatorname{Bil}(V), \psi(t)=\left\langle v, U_{t} w\right\rangle$ satisfies the KMS condition for $\beta=-1$ (which refers to a function on the strip $\{-1<\operatorname{Im} z<0\}$ ). ¿From [RvD77, Prop. 3.7] one can easily derive the implication (ii) $\Rightarrow$ (i) of Theorem 1.1 (cf. also [Lo08, Prop. 3.7]). In this case [RvD77, Thm. 3.8] even implies that $U_{t}=\Delta^{-i t / \beta}$ is the unique unitary one-parameter group satisfying the KMS condition for $\beta$. ¿From [RvD77, Thm. 3.9] one can also derive the implication (i) $\Rightarrow$ (ii). Instead of $\Delta$, Rieffel and van Daele work with the bounded operator $R=2(\mathbf{1}+\Delta)^{-1}$ which is the sum of the orthogonal projections of the real Hilbert space $\mathcal{H}$ onto the closed subspaces $V$ and $i V$. In our context this operator appears as $\mathbf{1}+i \widehat{C}$ for the skew-hermitian operator $\widehat{C}=i \frac{\Delta-\mathbf{1}}{\Delta+\mathbf{1}}$ (Lemma 4.2).

In the context of free fields, the interplay between standard real subspaces and von Neumann algebras of operators on Fock space has already been studied by Araki Ar63 and Eckmann/Osterwalder EO73. We refer to Yn94 for some particularly interesting concrete subspaces corresponding to fields on light rays and to [Ra00] for descriptions of standard real subspaces in terms of boundary values of holomorphic functions. Numerical aspects of the KMS condition and rather general holomorphic extension aspects have recently been studied in dMV16.

Notation: We follow the "physics convention" that the scalar product $\langle\cdot, \cdot\rangle$ on a complex Hilbert space is linear in the second argument.

For a real vector space $V$, we write $\operatorname{Bil}(V)$ for the complex vector space of complex-valued bilinear forms $V \times V \rightarrow \mathbb{C}$. For $h \in \operatorname{Bil}(V)$, we write $\bar{h}$ for the pointwise complex conjugate and put $h^{\top}(v, w):=h(w, v)$ and $h^{*}:=\bar{h}^{\top}$. We say that $h$ is hermitian if $\bar{h}=h^{\top}$, which means that $\operatorname{Re} h$ is symmetric and $\operatorname{Im} h$ is skew-symmetric. We write $\operatorname{Herm}(V) \subseteq \operatorname{Bil}(V)$ for the real subspace of hermitian forms.

Every $h \in \operatorname{Bil}(V)$ extends canonically to a sesquilinear form on $V_{\mathbb{C}}$ (linear in the second argument)

$$
h_{\mathbb{C}}\left(v+i w, v^{\prime}+i w^{\prime}\right):=h\left(v, v^{\prime}\right)-i h\left(w, v^{\prime}\right)+i h\left(v, w^{\prime}\right)+h\left(w, w^{\prime}\right) .
$$

We may therefore identify $\operatorname{Bil}(V)$ with the $\operatorname{space} \operatorname{Sesq}\left(V_{\mathbb{C}}\right)$ of sesquilinear forms on the complex vector space $V_{\mathbb{C}}$. We write $\operatorname{Bil}^{+}(V) \subseteq \operatorname{Bil}(V)$ for the convex cone of all those bilinear forms $f$ for which the sesquilinear extension to $V_{\mathbb{C}} \times V_{\mathbb{C}}$ is positive semidefinite, i.e., for which $h$ defines a positive definite kernel on $V$. 


\section{Contents}

1 Introduction 1

2 Positive definite functions and KMS conditions 5

3 Form-valued reflection positive functions 11

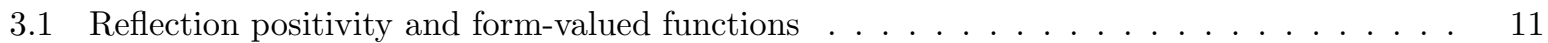

3.2 Reflection positivity for the trivial group . . . . . . . . . . . . . . . 14

3.3 Reflection positivity for the 2 -element group . . . . . . . . . . . . . 16

4 Reflection positive functions and KMS conditions 17

4.1 From form-valued to operator-valued functions $\ldots \ldots \ldots \ldots \ldots \ldots$

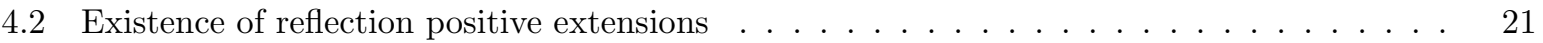

4.3 Integral representation of reflection positive functions . . . . . . . . . . . . . 23

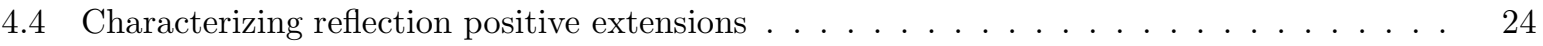

4.5 Realization by resolvents of the Laplacian . . . . . . . . . . . . . . . . . . 27

5 The case $\beta=\infty \quad 29$

A Some background on positive definite kernels 31

A.1 Form-valued positive definite kernels . . . . . . . . . . . . . . . . . . . . . 31

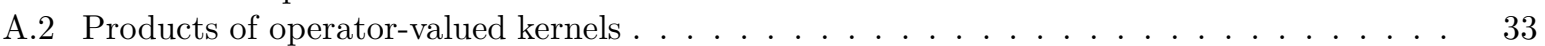

A.3 From real to complex-valued kernels . . . . . . . . . . . . . . . . . . . . 33

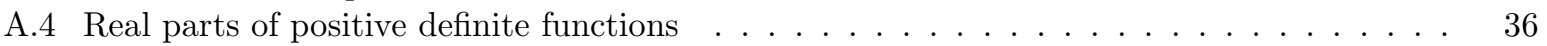

B Standard real subspaces via contractions

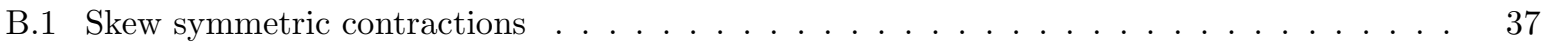

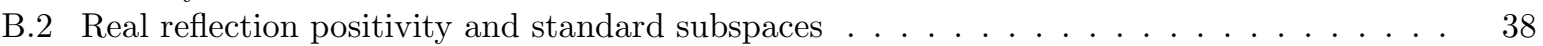

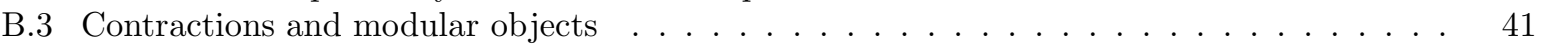

\section{Positive definite functions and KMS conditions}

Throughout this section $V$ is an arbitrary real vector space. We recall from Definition A.3 that a function $\psi: \mathbb{R} \rightarrow \operatorname{Bil}(V)$ is called positive definite if the kernel $K((t, v),(s, w)):=\psi(t-s)(v, w)$ on $\mathbb{R} \times V$ is positive definite. The main result of this section is Theorem 2.6. which is Theorem 1.1 from the introduction. This result leads in particular to the analytic continuation of $\psi$ to the strip $\mathcal{S}_{\beta}$. We also explain how the corresponding representation of $\mathbb{R}$ can be realized in a Hilbert space consisting of holomorphic functions on the strip $\mathcal{S}_{\beta / 2}$ with continuous boundary values (Proposition 2.9).

We call a function $\psi: \overline{\mathcal{S}_{\beta}} \rightarrow \operatorname{Bil}(V)$ pointwise continuous if, for all $v, w \in V$, the function $\psi^{v, w}(z):=$ $\psi(z)(v, w)$ is continuous. Moreover, we say that $\psi$ is pointwise holomorphic in $\mathcal{S}_{\beta}$, if, for all $v, w \in V$, the function $\left.\psi^{v, w}\right|_{\mathcal{S}_{\beta}}$ is holomorphic. By the Schwarz reflection principle, any pointwise continuous pointwise holomorphic function $\psi$ is uniquely determined by its restriction to $\mathbb{R}$. 
Definition 2.1. For $\beta>0$, let $\mathcal{S}_{\beta}:=\{z \in \mathbb{C}: 0<\operatorname{Im} z<\beta\}$. For a real vector space $V$, we say that a positive definite function $\psi: \mathbb{R} \rightarrow \operatorname{Bil}(V)$ satisfies the $K M S$ condition for $\beta>0$ if $\psi$ extends to a function $\psi: \overline{\mathcal{S}_{\beta}} \rightarrow \operatorname{Bil}(V)$ which is pointwise continuous, pointwise holomorphic on $\mathcal{S}_{\beta}$, and satisfies

$$
\psi(i \beta+t)=\overline{\psi(t)} \quad \text { for } \quad t \in \mathbb{R} .
$$

Lemma 2.2. Suppose that $\psi: \mathbb{R} \rightarrow \operatorname{Bil}(V)$ satisfies the $K M S$ condition for $\beta>0$. Then

$$
\psi(-\bar{z})=\psi(z)^{*} \quad \text { and } \quad \psi(i \beta+\bar{z})=\overline{\psi(z)} \quad \text { for } \quad z \in \overline{\mathcal{S}_{\beta}} .
$$

The function $\varphi:[0, \beta] \rightarrow \operatorname{Bil}(V), \varphi(t):=\psi(i t)$ has hermitian values and satisfies

$$
\varphi(\beta-t)=\overline{\varphi(t)} \quad \text { for } \quad 0 \leq t \leq \beta .
$$

It extends to a unique pointwise continuous symmetric $2 \beta$-periodic function $\varphi: \mathbb{R} \rightarrow \operatorname{Herm}(V)$ satisfying

$$
\varphi(\beta+t)=\overline{\varphi(t)} \quad \text { for } \quad t \in \mathbb{R} .
$$

Proof. Note that $\psi(-t)=\psi(t)^{*}$ holds for every positive definite function $\psi: \mathbb{R} \rightarrow \operatorname{Bil}(V)$. By analytic continuation (resp., the Schwarz reflection principle), this leads to the first part of (4). Likewise, condition (3) leads to the second part of (4). This in turn implies (5), and the remainder is clear.

Remark 2.3. Note that (4) implies in particular that $\psi(i \beta / 2+t)$ is real-valued for $t \in \mathbb{R}$ (cf. RvD77, Prop. 3.5]).

We now introduce standard real subspaces $V \subseteq \mathcal{H}$ and the associated modular objects $(\Delta, J)$.

Definition 2.4. A closed real subspace $V$ of a complex Hilbert space $\mathcal{H}$ is said to be standard if

$$
V \cap i V=\{0\} \quad \text { and } \quad \overline{V+i V}=\mathcal{H} .
$$

For every standard real subspace $V \subseteq \mathcal{H}$, we define an unbounded antilinear operator

$$
S: \mathcal{D}(S)=V+i V \rightarrow \mathcal{H}, \quad S(v+i w):=v-i w, \quad v, w \in V .
$$

Then $S$ is closed and has a polar decomposition $S=J \Delta^{1 / 2}$, where $J$ is an anti-unitary involution and $\Delta$ a positive selfadjoint operator (cf. [NÓ15, Lemma 4.2]; see also [BR02, Prop. 2.5.11], [Lo08, Prop. 3.3]). We call $(\Delta, J)$ the modular objects of $V$.

Remark 2.5. (a) From $S^{2}=$ id, it follows that the modular objects $(\Delta, J)$ of a standard real subspace satisfies the modular relation

$$
J \Delta=\Delta^{-1} .
$$

If, conversely, $(\Delta, J)$ is a pair of a positive selfadjoint operator $\Delta$ and an antilinear involution $J$ satisfying (6), then $S:=J \Delta^{1 / 2}$ is an unbounded antilinear involution with $\mathcal{D}(S)=\mathcal{D}\left(\Delta^{1 / 2}\right)$ whose fixed point space $\operatorname{Fix}(S)$ is a standard real subspace. Thus standard real subspaces are parametrized by pairs $(\Delta, J)$ satisfying ([6) (cf. Lo08, Prop. 3.2] and [NÓ15, Lemma 4.4]).

(b) As the unitary one-parameter group $\Delta^{i t}$ commutes with $J$ and $\Delta$, it leaves the real subspace $V=\operatorname{Fix}(S)$ invariant.

We now come to the proof of Theorem 1.1 from the introduction. 
Theorem 2.6. (Characterization of the KMS condition) Let $V$ be a real vector space and let $\psi: \mathbb{R} \rightarrow$ $\operatorname{Bil}(V)$ be a pointwise continuous positive definite function. Then the following are equivalent:

(i) $\psi$ satisfies the $K M S$ condition for $\beta>0$.

(ii) There exists a standard real subspace $V_{1}$ in a Hilbert space $\mathcal{H}$ and a linear map $j: V \rightarrow V_{1}$ such that

$$
\psi(t)(v, w)=\left\langle j(v), \Delta^{-i t / \beta} j(w)\right\rangle \quad \text { for } \quad t \in \mathbb{R}, v, w \in V .
$$

(iii) There exists a $\operatorname{Bil}^{+}(V)$-valued regular Borel measure $\mu$ on $\mathbb{R}$ satisfying $d \mu(-\lambda)=e^{-\beta \lambda} d \bar{\mu}(\lambda)$, such that

$$
\psi(t)=\int_{\mathbb{R}} e^{i t \lambda} d \mu(\lambda)=\widehat{\mu}(t)
$$

If these conditions are satisfied, then the function $\psi: \overline{\mathcal{S}_{\beta}} \rightarrow \operatorname{Bil}(V)$ is pointwise bounded.

Proof. (i) $\Rightarrow$ (ii): From the GNS construction (PropositionA.4) we obtain a continuous unitary representation $(U, \mathcal{H})$ and a linear map $j: V \rightarrow \mathcal{H}$ such that

$$
\psi(t)(v, w)=\left\langle j(v), U_{t} j(w)\right\rangle \quad \text { for } \quad t \in \mathbb{R}, v, w \in V .
$$

We further assume that the range of the map

$$
\zeta: \mathbb{R} \times V \rightarrow \mathcal{H}, \quad \zeta(t, v):=U_{t} j(v)
$$

spans a dense subspace. Using Stone's Theorem, we write $U_{t}=e^{-i t H}$ for a selfadjoint operator $H$ on $\mathcal{H}$ and consider the positive selfadjoint operator

$$
\Delta:=e^{\beta H} \quad \text { satisfying } \quad U_{t}=\Delta^{-i t / \beta} \quad \text { for } \quad t \in \mathbb{R} .
$$

With the $B(\mathcal{H})$-valued spectral measure $P$ on $\mathbb{R}$ with $H=\int_{\mathbb{R}} \lambda d P(\lambda)$, we thus obtain

$$
\psi(t)(v, w)=\left\langle j(v), e^{-i t H} j(w)\right\rangle=\int_{\mathbb{R}} e^{-i t \lambda} d P^{j(v), j(w)}(\lambda),
$$

where $P^{v, w}=\langle v, P(\cdot) w\rangle$. The KMS condition for $\psi$ entails that, for each $v \in V$, the function $\psi(t)(v, v)$ extends holomorphically to $\overline{\mathcal{S}_{\beta}}$, which entails that the integral $\int_{\mathbb{R}} e^{\beta \lambda} d P^{j(v), j(v)}(\lambda)$ is finite, and hence that $j(V) \subseteq \mathcal{D}\left(\Delta^{1 / 2}\right)$ (NÓ15, Lemma B.4]). The uniqueness of analytic continuation (Schwarz' principle) now implies that

$$
\psi(x+i y)(v, w)=\int_{\mathbb{R}} e^{-i(x+i y) \lambda} d P^{j(v), j(w)}(\lambda)=\left\langle\Delta^{y / 2 \beta} j(v), \Delta^{-i x / \beta} \Delta^{y / 2 \beta} j(w)\right\rangle
$$

for $v, w \in V$ and $0 \leq y \leq \beta$. Since $\mathcal{D}\left(\Delta^{1 / 2}\right)$ is $U$-invariant, we obtain from the KMS condition

$$
\begin{aligned}
\left\langle\Delta^{1 / 2} \zeta(t, v), \Delta^{1 / 2} \zeta(s, w)\right\rangle & =\left\langle\Delta^{1 / 2} j(v), \Delta^{1 / 2} U_{s-t} j(w)\right\rangle=\psi(i \beta+s-t)(v, w) \\
& =\overline{\psi(s-t)(v, w)}=\overline{\langle\zeta(t, v), \zeta(s, w)\rangle} .
\end{aligned}
$$

This implies the existence of a unique antilinear isometry $J: \mathcal{H} \rightarrow \mathcal{H}$ with

$$
J \zeta(t, v)=\Delta^{1 / 2} \zeta(t, v) \quad \text { for all } \quad t \in \mathbb{R}, v \in V .
$$


Then

$$
U_{s} J \zeta(t, v)=\Delta^{1 / 2} \zeta(t+s, v)=J \zeta(t+s, v)=J U_{s} \zeta(t, v) \quad \text { for } \quad t, s \in \mathbb{R}, v \in V
$$

shows that $J$ commutes with every $U_{t}$. This implies that $J \Delta^{1 / 2} J^{-1}=\Delta^{-1 / 2}$, so that

$$
\zeta(t, v)=J^{-1} \Delta^{1 / 2} \zeta(t, v)=\Delta^{-1 / 2} J^{-1} \zeta(t, v),
$$

which in turn implies

$$
J \zeta(t, v)=\Delta^{1 / 2} \zeta(t, v)=J^{-1} \zeta(t, v) \quad \text { for } \quad t \in \mathbb{R}, v \in V .
$$

Since the range of $\zeta$ is total, it follows that $J^{-1}=J$, so that $J$ is an anti unitary involution. Therefore $(\Delta, J)$ is the modular objects of the standard real subspace $V_{1}:=\operatorname{Fix}(S)$ for the unbounded antilinear involution $S:=J \Delta^{1 / 2}$ (Remark 2.5) .

For $v \in V$ we now have $j(v) \in \mathcal{D}(S)=\mathcal{D}\left(\Delta^{1 / 2}\right)$ and $S j(v)=J \Delta^{1 / 2} j(v)=J^{2} j(v)=j(v)$, so that $j(V) \subseteq V_{1}$. This completes the proof of (ii).

(ii) $\Rightarrow$ (iii): For $v, w \in V$ we have

$$
\psi(t)(v, w)=\left\langle j(v), \Delta^{-i t / \beta} j(w)\right\rangle=\int_{\mathbb{R}} e^{i t \lambda}\langle j(v), d P(\lambda) j(w)\rangle,
$$

where $P$ is the spectral measure of the selfadjoint operator $L:=-\frac{1}{\beta} \log \Delta$ (the Liouvillean). We therefore consider the $\mathrm{Bil}^{+}(V)$-valued measure defined by

$$
\mu(\cdot)(v, w):=\langle j(v), P(\cdot) j(w)\rangle=P^{j(v), j(w)} .
$$

It remains to show that $d \mu(-\lambda)=e^{-\beta \lambda} d \bar{\mu}(\lambda)$, which means that $r_{*} \mu=e_{-\beta} \bar{\mu}$ holds for $r(\lambda)=-\lambda$. To verify this relation, we first observe that $J L J=-L$ implies that $J P J=r_{*} P$. This leads to

$$
\begin{aligned}
\overline{\mu(\cdot)(v, w)} & =\langle P(\cdot) j(w), j(v)\rangle=\langle P(\cdot) S j(w), S j(v)\rangle=\left\langle P(\cdot) J \Delta^{1 / 2} j(w), J \Delta^{1 / 2} j(v)\right\rangle \\
& =\left\langle J P(\cdot) J \Delta^{1 / 2} j(v), \Delta^{1 / 2} j(w)\right\rangle=\left\langle\left(r_{*} P\right)(\cdot) \Delta^{1 / 2} j(v), \Delta^{1 / 2} j(w)\right\rangle \\
& =e_{\beta} \cdot\left\langle\left(r_{*} P\right)(\cdot) j(v), j(w)\right\rangle=e_{\beta} \cdot\left(r_{*} \mu\right)(\cdot)(v, w) .
\end{aligned}
$$

This implies that $\bar{\mu}=e_{\beta} \cdot r_{*} \mu$.

(iii) $\Rightarrow$ (i): Condition (iii) implies that $\psi(0)=\mu(\mathbb{R})$ exists, so that $\mu$ is a pointwise finite measure. Further, the relation $r_{*} \mu=e_{-\beta} \bar{\mu}$ implies that the measure $e_{-\beta} \mu$ is also finite. Therefore the integral

$$
\psi(z):=\int_{\mathbb{R}} e^{i z \lambda} d \mu(\lambda)
$$

exists pointwise and extends $\psi$ to $\overline{\mathcal{S}_{\beta}}$ in such a way that this extension is pointwise continuous on $\overline{\mathcal{S}_{\beta}}$ and pointwise holomorphic on the interior. The relation $r_{*} \mu=e_{-\beta} \bar{\mu}$ further leads to

$$
\psi(i \beta+t)=\int_{\mathbb{R}} e^{\lambda(-\beta+i t)} d \mu(\lambda)=\int_{\mathbb{R}} e_{-\beta}(\lambda) e^{i \lambda t} d \mu(\lambda)=\int_{\mathbb{R}} e^{i \lambda t} d\left(r_{*} \bar{\mu}\right)(\lambda)=\int_{\mathbb{R}} e^{-i \lambda t} d \bar{\mu}(\lambda)=\overline{\psi(t)} .
$$

Therefore $\psi$ satisfies the KMS condition for $\beta$.

We finally assume that (i)-(iii) are satisfied and show that $\psi$ is pointwise bounded on $\overline{\mathcal{S}_{\beta}}$. Since each $\psi(z)$ extends to a sesquilinear form $\psi(z)_{\mathbb{C}}$ on $V_{\mathbb{C}}$, in view of the Polarization Identity, it suffices to show the 
boundedness of the functions $z \mapsto \psi(z)_{\mathbb{C}}(v, v)$ for $v \in V_{\mathbb{C}}$. For the positive measure $\mu^{v, v}(E):=\mu(E)_{\mathbb{C}}(v, v)$, we obtain from (9) the estimate

$$
\left|\psi(z)_{\mathbb{C}}(v, v)\right| \leq \int_{\mathbb{R}}\left|e^{-i \lambda z}\right| d \mu^{v, v}(\lambda)=\int_{\mathbb{R}} e^{\lambda \operatorname{Im} z} d \mu^{v, v}(\lambda) .
$$

The convexity of the function on the right, the Laplace transform of the finite positive measure $\mu^{v, v}$, and $\psi(\beta i)(v, v)=\left\|\Delta^{1 / 2} j(v)\right\|^{2}<\infty$ now imply the boundedness of $\psi(z)_{\mathbb{C}}(v, v)$.

Remark 2.7. An important special case arise from a $C^{*}$-dynamical system $(\mathcal{A}, \mathbb{R}, \alpha)$ for $V:=\mathcal{A}_{h}:=$ $\left\{A \in \mathcal{A}: A^{*}=A\right\}$ and an invariant state $\omega$ on $\mathcal{A}$. Such a state is a $\beta$-KMS state if and only if

$$
\psi: \mathbb{R} \rightarrow \operatorname{Bil}\left(\mathcal{A}_{h}\right), \quad \psi(t)(A, B):=\omega\left(A \alpha_{t}(B)\right)
$$

satisfies the KMS condition for $\beta>0$ (cf. [NÓ15, Prop. 5.2], [RvD77, Thm. 4.10], [BR96]). If $\left(\pi_{\omega}, U^{\omega}, \mathcal{H}_{\omega}, \Omega\right)$ is the corresponding covariant GNS representation of $(\mathcal{A}, \mathbb{R})$, then

$$
\omega(A)=\left\langle\Omega, \pi_{\omega}(A) \Omega\right\rangle \quad \text { for } \quad A \in \mathcal{A} \quad \text { and } \quad U_{t}^{\omega} \Omega=\Omega \quad \text { for } \quad t \in \mathbb{R} .
$$

Therefore

$$
\psi(t)(A, B)=\omega\left(A \alpha_{t}(B)\right)=\left\langle\Omega, \pi_{\omega}\left(A \alpha_{t}(B)\right) \Omega\right\rangle=\left\langle\Omega, \pi_{\omega}(A) U_{t}^{\omega} \pi_{\omega}(B) U_{-t}^{\omega} \Omega\right\rangle=\left\langle\pi_{\omega}(A) \Omega, U_{t}^{\omega} \pi_{\omega}(B) \Omega\right\rangle
$$

for $A, B \in \mathcal{A}_{h}$. The corresponding standard real subspace of $\mathcal{H}_{\omega}$ is $V_{1}:=\overline{\pi_{\omega}\left(\mathcal{A}_{h}\right) \Omega}$.

Corollary 2.8. If $\psi: \mathbb{R} \rightarrow \operatorname{Bil}(V)$ satisfies the $\beta$-KMS condition, then the kernel

$$
K: \overline{\mathcal{S}_{\beta / 2}} \times \overline{\mathcal{S}_{\beta / 2}} \rightarrow \operatorname{Bil}(V), \quad K(z, w)(\xi, \eta):=\psi(z-\bar{w})(\xi, \eta)
$$

is positive definite.

Proof. This follows immediately from the following relation that we derive from (8):

$$
K(z, w)(\xi, \eta)=\psi(z-\bar{w})(\xi, \eta)=\left\langle\Delta^{-\frac{i \bar{z}}{\beta}} j(\xi), \Delta^{-\frac{i \bar{w}}{\beta}} j(\eta)\right\rangle \quad \text { for } \quad \xi, \eta \in V, z, w \in \overline{\mathcal{S}_{\beta / 2}}
$$

Now that we know from Corollary 2.8 that the kernel $K$ in (10) is positive definite, we obtain a corresponding reproducing kernel Hilbert space consisting of functions on $\overline{\mathcal{S}_{\beta / 2}} \times V$ which are linear in the second argument and holomorphic on $\mathcal{S}_{\beta / 2}$ in the first. We may therefore think of these functions as having values in the algebraic dual space $V^{*}:=\operatorname{Hom}(V, \mathbb{R})$ of $V$. We write $\mathcal{O}\left(\overline{\mathcal{S}_{\beta / 2}}, V^{*}\right)$ for the space of those functions $f: \overline{\mathcal{S}_{\beta / 2}} \rightarrow V^{*}$ with the property that, for every $\eta \in V$, the function $z \mapsto f(z)(\eta)$ is continuous on $\overline{\mathcal{S}_{\beta / 2}}$ and holomorphic on the open strip $\mathcal{S}_{\beta / 2}$.

Proposition 2.9. (Realization of $\mathcal{H}_{\psi}$ on $\mathcal{O}\left(\overline{\mathcal{S}_{\beta / 2}}, V^{*}\right)$ ) Assume that $\psi: \mathbb{R} \rightarrow \operatorname{Bil}(V)$ satisfies the KMS condition for $\beta>0$ and let $\psi: \overline{\mathcal{S}_{\beta}} \rightarrow \operatorname{Bil}(V)$ denote the corresponding extension and $\mathcal{H}_{\psi} \subseteq \mathcal{O}\left(\overline{\mathcal{S}_{\beta / 2}}, V^{*}\right)$ denote the Hilbert space with reproducing kernel

$$
K(z, w)(\xi, \eta):=\psi(z-\bar{w})(\xi, \eta) \quad \text { for } \quad \xi, \eta \in V
$$

i.e.,

$$
f(z)(\xi)=\left\langle K_{z, \xi}, f\right\rangle \quad \text { for } \quad f \in \mathcal{H}_{\psi}, \quad \text { where } \quad K_{z, \xi}(w)(\eta)=\psi(w-\bar{z})(\eta, \xi)
$$


Then

$$
\left(U_{t}^{\psi} f\right)(z):=f(z+t), \quad t \in \mathbb{R}, z \in \overline{\mathcal{S}_{\beta / 2}}
$$

defines a unitary one-parameter group on $\mathcal{H}_{\psi}$,

$$
j: V \rightarrow \mathcal{H}_{\psi}, \quad j(\eta)(z):=\psi(z)(\cdot, \eta)
$$

is a linear map with $U^{\psi}$-cyclic range, and

$$
\psi(t)(\xi, \eta)=\left\langle j(\xi), U_{t}^{\psi} j(\eta)\right\rangle \quad \text { for } \quad t \in \mathbb{R}, \xi, \eta \in V .
$$

The anti-unitary involution on $\mathcal{H}_{\psi}$ corresponding to the standard real subspace $V_{1} \subseteq \mathcal{H}_{\psi}$ from Theorem2.6 is given by

$$
\left(J_{1} f\right)(z):=\overline{f\left(\bar{z}+\frac{i \beta}{2}\right)} .
$$

Proof. First we recall that the natural reproducing kernel Hilbert space $\mathcal{H}_{\psi}=\mathcal{H}_{K}$ is generated by function $K_{(w, \eta)}$ satisfying

$$
K_{(w, \eta)}(z)(\xi)=\left\langle K_{(z, \xi)}, K_{(w, \eta)}\right\rangle=K(z, w)(\xi, \eta)=\psi(z-\bar{w})(\xi, \eta) .
$$

As a function of $z$, the kernel $K$ is continuous on $\overline{\mathcal{S}_{\beta / 2}}$ and holomorphic on the interior. Therefore $\mathrm{Ne00}$, Prop. I.1.9] implies that $\mathcal{H}_{\psi}$ is a subspace of $\mathcal{O}\left(\overline{\mathcal{S}_{\beta / 2}}, V^{*}\right)$, where, for every $f \in \mathcal{H}_{\psi}$ and $\xi \in V$, we have

$$
f(z)(\xi)=\left\langle K_{(z, \xi)}, f\right\rangle .
$$

That the formula for $U_{t}^{\psi}$ defines a unitary one-parameter group on $\mathcal{H}_{\psi}$ follows directly from the invariance of the kernel $K$ under the action of $\mathbb{R}$ on $\overline{\mathcal{S}_{\beta}}$ by translation.

Next we observe that

$$
\left\langle j(\xi), U_{t}^{\psi} j(\eta)\right\rangle=\left\langle K_{(0, \xi)}, U_{t}^{\psi} K_{(0, \eta)}\right\rangle=\left\langle K_{(0, \xi)}, K_{(-t, \eta)}\right\rangle=\psi(t)(\xi, \eta) .
$$

To see that $j(V)$ is $U^{\psi}$-cyclic, we have to show that the elements $U_{t}^{\psi} j(\eta)=K_{(-t, \eta)}$ form a total subset of $\mathcal{H}_{\psi}$. This means that any $f \in \mathcal{H}_{\psi}$ with $0=\left\langle K_{(t, \eta)}, f\right\rangle=f(t)(\eta)$ for every $t \in \mathbb{R}$ and $\eta \in V$ vanishes. As the function $t \mapsto f(t)(\eta)$ extends to a continuous function on $\overline{\mathcal{S}_{\beta / 2}}$, holomorphic on the interior, it vanishes by the Schwarz Reflection Principle. Further, $\eta$ was arbitrary, so that $f=0$ follows.

Now we turn to the involution $J_{1}$. As $K_{(w, \eta)}(z)=\psi(z-\bar{w})(\cdot, \eta)$, the operator $J_{1}$ on $\mathcal{O}\left(\overline{\mathcal{S}_{\beta / 2}}, V^{*}\right)$, defined by the right hand side of (11) satisfies

$$
\begin{aligned}
\left(J_{1} K_{(w, \eta)}\right)(z) & =\overline{K_{(w, \eta)}\left(\bar{z}+\frac{i \beta}{2}\right)}=\overline{\psi\left(\bar{z}+\frac{i \beta}{2}-\bar{w}\right)(\cdot, \eta)}=\psi\left(i \beta+z-\frac{i \beta}{2}-w\right)(\cdot, \eta) \\
& =\psi\left(z+\frac{i \beta}{2}-w\right)(\cdot, \eta)=K_{(\bar{w}+i \beta / 2, \eta)}(z) .
\end{aligned}
$$

Here we have used that $\overline{\psi(z)}=\psi(i \beta+\bar{z})$ (Lemma 2.2). ¿From

$$
\begin{aligned}
\left\langle K_{(\bar{w}+i \beta / 2, \eta)}, K_{(\bar{z}+i \beta / 2, \xi)}\right\rangle & =\overline{K(\bar{z}+i \beta / 2, \bar{w}+i \beta / 2)(\xi, \eta)}=\overline{\psi(i \beta+\bar{z}-w)(\xi, \eta)} \\
& =\psi(z-\bar{w})(\xi, \eta)=\left\langle K_{(z, \xi)}, K_{(w, \eta)}\right\rangle
\end{aligned}
$$


it now follows that the operator $J_{1}$ in (11) leaves the subspace $\mathcal{H}_{\psi}$ invariant and defines an antilinear isometry on this space. From the explicit formula it follows that $J_{1}$ is an involution. It is also clear that $J_{1}$ commutes with the unitary operators $\left(U_{t} f\right)(z)=f(z+t)$.

The relation $U_{t} K_{(w, \eta)}=K_{(w-t, \eta)}$ leads by analytic continuation to

$$
J_{1} K_{(0, \eta)}=K_{(i \beta / 2, \eta)}=\Delta^{1 / 2} K_{(0, \eta)} .
$$

In the proof of Theorem [2.6, we have seen that, for $\eta \in V$ and $t \in \mathbb{R}$, the anti-unitary involution $J$ corresponding to the associated standard real subspace $V_{1}$ satisfies

$$
J j(\eta)=\Delta^{1 / 2} j(\eta) .
$$

As both, $J$ and $J_{1}$ commute with every $U_{t}$ and the subset $\left\{U_{t} j(\eta): t \in \mathbb{R}, \eta \in V\right\}$ is total in $\mathcal{H}_{\psi}$, we conclude that $J_{1}=J$.

\section{Form-valued reflection positive functions}

In this section we discuss reflection positivity on the level of form-valued positive definite function. This is particularly well-adapted to reflection positive Hilbert spaces $\left(\mathcal{E}, \mathcal{E}_{+}, \theta\right)$, for which $\mathcal{E}_{+}$is generated by elements of the form $U_{g}^{-1} j(v)$, where $g$ is contained in a certain subset $G_{+} \subseteq G$ which is not necessarily a subsemigroup, and $j: V \rightarrow \mathcal{H}$ is a linear map for which $U_{G} j(V)$ spans a dense subspace of $\mathcal{E}$. In particular, we present a version of the GNS construction in this context (Proposition 3.9) and we briefly discuss it more specifically for the trivial group $G=\{\mathbf{1}\}$ (Subsection 3.2) and the 2-element group (Subsection 3.3). The latter case shows explicitly that the cone of reflection positive functions does not adapt naturally to the decomposition into even and odd functions. Put differently, if a reflection positive representation decomposes into two subrepresentations, the summands need not be reflection positive (see also [NÓ14a]).

\subsection{Reflection positivity and form-valued functions}

Let $(G, \tau)$ be a symmetric Lie group, i.e., $G$ is a Lie group and $\tau \in \operatorname{Aut}(G)$ with $\tau^{2}=\operatorname{id}_{G}$. In the following we write $G_{\tau}:=G \rtimes\{\mathbf{1}, \tau\}$ and $g^{\sharp}:=\tau(g)^{-1}$ ([NÓ14a] $)$. In this section we introduce reflection positive functions on $G_{\tau}$ with values in $\operatorname{Bil}(V)$ for a real vector space $V$.

Definition 3.1. Let $\mathcal{E}$ be a Hilbert space and let $\theta \in \mathrm{U}(\mathcal{E})$ be an involution. A closed subspace $\mathcal{E}_{+} \subseteq \mathcal{E}$ is called $\theta$-positive if $\langle\theta v, v\rangle \geq 0$ for $v \in \mathcal{E}_{+}$. We then call the triple $\left(\mathcal{E}, \mathcal{E}_{+}, \theta\right)$ a reflection positive Hilbert space. For a reflection positive Hilbert space we put $\mathcal{N}:=\left\{v \in \mathcal{E}_{+}:\langle\theta v, v\rangle=0\right\}$ and write $q: \mathcal{E}_{+} \rightarrow \mathcal{E}_{+} / \mathcal{N}, v \mapsto \widehat{v}=q(v)$ for the quotient map and $\widehat{\mathcal{E}}$ for the Hilbert completion of $\mathcal{E}_{+} / \mathcal{N}$ with respect to the norm $\|\widehat{v}\|_{\widehat{\mathcal{E}}}:=\|\widehat{v}\|:=\sqrt{\langle\theta v, v\rangle}$.

Example 3.2. Suppose that $K: X \times X \rightarrow \mathbb{C}$ is a positive definite kernel on the set $X$ and that $\tau: X \rightarrow X$ is an involution leaving $K$ invariant. We further assume that $X_{+} \subseteq X$ is a subset with the property that the kernel $K^{\tau}(x, y):=K(\tau x, y)$ is also positive definite on $X_{+}$.

Let $\mathcal{E}:=\mathcal{H}_{K} \subseteq \mathbb{C}^{X}$ denote the corresponding reproducing kernel Hilbert space generated by elements $\left(K_{x}\right)_{x \in X}$ with $\left\langle K_{x}, K_{y}\right\rangle=K(x, y)$. Then the closed subspace $\mathcal{E}_{+} \subseteq \mathcal{E}$ generated by $\left(K_{x}\right)_{x \in X_{+}}$is $\theta$-positive for $(\theta f)(x):=f(\tau x)$. We thus obtain a reflection positive Hilbert space $\left(\mathcal{E}, \mathcal{E}_{+}, \theta\right)$. We call such kernels $K$ reflection positive with respect to $\left(X, X_{+}, \tau\right)$. 
Definition 3.3. Let $G_{+} \subseteq G$ be a subset. Let $V$ be a real vector space and let $j: V \rightarrow \mathcal{H}$ be a linear map whose range is cyclic for the unitary representation $(U, \mathcal{E})$ of $G_{\tau}$. Then we say that $(U, \mathcal{E}, j, V)$ is reflection positive with respect to $G_{+} \subseteq G$ if, for $\mathcal{E}_{+}:=\overline{\operatorname{span} U_{G_{+}}^{-1} j(V)}$, the triple $\left(\mathcal{E}, \mathcal{E}_{+}, U_{\tau}\right)$ is a reflection positive Hilbert space.

Definition 3.4. Let $V$ be a real vector space. We call a function $\varphi: G_{\tau} \rightarrow \operatorname{Bil}(V)$ reflection positive with respect to the subset $G_{+}$of $G$ if

$(\mathrm{RP} 1) \varphi$ is positive definite and

(RP2) the kernel $(s, t) \mapsto \varphi\left(s t^{\sharp} \tau\right)=\varphi\left(s \tau t^{-1}\right)$ is positive definite on $G_{+}$.

Remark 3.5. Let $\varphi: G_{\tau} \rightarrow \operatorname{Bil}(V)$ be a positive definite function, so that the kernel $K((x, v),(y, w)):=$ $\varphi\left(x y^{-1}\right)(v, w)$ on $G_{\tau} \times V$ is positive definite. The involution $\tau$ acts on $G_{\tau} \times V$ by $\tau \cdot(g, v):=(g \tau, v)$ and the corresponding kernel $K^{\tau}((x, v),(y, w)):=K((x \tau, v),(y, w))=\varphi\left(x \tau y^{-1}\right)(v, w)$ is positive definite on $G_{+} \times V$ if and only if $\varphi$ is reflection positive in the sense of Example 3.2.

Positive definite functions on $G$ extend canonically to $G_{\tau}$ if they are $\tau$-invariant:

Lemma 3.6. Let $V$ be a real vector space and let $(G, \tau)$ be a symmetric Lie group. Then the following assertions hold:

(i) If $\varphi: G \rightarrow \operatorname{Bil}(V)$ is a positive definite function which is $\tau$-invariant in the sense that $\varphi \circ \tau=\varphi$, then $\widehat{\varphi}(g, \tau):=\varphi(g)$ defines an extension to $G_{\tau}$ which is positive definite and $\tau$-biinvariant.

(ii) Let $(U, \mathcal{H})$ be a unitary representation of $G_{\tau}$, let $\theta:=U_{\tau}$, let $j: V \rightarrow \mathcal{H}$ be a linear map, and let $\varphi(g)(v, w)=\left\langle j(v), U_{g} j(w)\right\rangle$ be the corresponding $\operatorname{Bil}(V)$-valued positive definite function. Then the following are equivalent:

(a) $\theta j(v)=j(v)$ for every $v \in V$.

(b) $\varphi$ is $\tau$-biinvariant.

(c) $\varphi$ is left $\tau$-invariant.

Proof. (i) From the GNS construction (Proposition A.4), we obtain a continuous unitary representation $(U, \mathcal{H})$ of $G$ and a linear map $j: V \rightarrow \mathcal{H}$ such that

$$
\varphi(g)(v, w)=\left\langle j(v), U_{g} j(w)\right\rangle \quad \text { for } \quad g \in G, v, w \in V .
$$

As $\varphi(g)(v, w)=\varphi(\tau(g))(v, w)$, the uniqueness in the GNS construction provide a unitary operator $\theta: \mathcal{H} \rightarrow \mathcal{H}$ with

$$
\theta U_{g} j(v)=U_{\tau(g)} j(v) \quad \text { for } \quad g \in G, v \in V .
$$

Note that $\theta$ fixes each $j(v)$. Therefore $U_{\tau}:=\theta$ defines an extension of $G$ to a unitary representation of $G_{\tau}$ on $\mathcal{H}$. Hence $\psi(g)(v, w)=\left\langle j(v), U_{g} j(w)\right\rangle$ defines a positive definite $\operatorname{Bil}(V)$-valued function on $G_{\tau}$ which satisfies

$$
\psi(g, \tau)(v, w)=\left\langle\theta j(v), U_{g} j(w)\right\rangle=\left\langle j(v), U_{g} j(w)\right\rangle=\varphi(g)(v, w) \quad \text { for } \quad g \in G, v, w \in V .
$$

(ii) Clearly, (a) $\Rightarrow$ (b) $\Rightarrow$ (c). It remains to show that (c) implies (a). So we assume that $\varphi(\tau g)=\varphi(g)$ for $g \in G_{\tau}$. This means that, for every $v, w \in V$, we have

$$
\left\langle j(v), U_{g} j(w)\right\rangle=\varphi(g)(v, w)=\varphi(\tau g)(v, w)=\left\langle j(v), \theta U_{g} j(w)\right\rangle=\left\langle\theta j(v), U_{g} j(w)\right\rangle .
$$

Since $U_{G_{\tau}} j(V)$ is total in $\mathcal{H}$, this implies that $\theta j(v)=j(v)$ for every $v \in V$. 
Remark 3.7. (a) As $G_{\tau}$ consists of the two cosets $G$ and $G \tau=G \times\{\tau\}$, every function $\varphi$ on $G_{\tau}$ is given by a pair of functions on $G$ :

$$
\varphi_{ \pm}: G \rightarrow \operatorname{Bil}(V), \quad \varphi_{+}(g):=\varphi(g, \mathbf{1}), \quad \varphi_{-}(g):=\varphi(g, \tau) .
$$

Then (RP2) is a condition on $\varphi_{-}$alone, and (RP1) is a condition on the pair $\left(\varphi_{+}, \varphi_{-}\right)$.

(b) If $\varphi$ is reflection positive, then its complex conjugate $\bar{\varphi}$ is also reflection positive because the convex cone of positive definite kernels on a set is stable under complex conjugation. This implies in particular that $\operatorname{Re} \varphi=\frac{1}{2}(\varphi+\bar{\varphi})$ is reflection positive (cf. Theorem A.13).

The following lemma provides a tool which is sometimes convenient to verify positive definiteness of a function on the extended group $G_{\tau}$ in terms of a kernel on the original group $G$.

Lemma 3.8. Every function $\varphi: G_{\tau} \rightarrow B(V)$ leads to a $M_{2}(B(V))$-valued kernel

$$
Q: G \times G \rightarrow M_{2}(B(V)) \cong B(V \oplus V), \quad Q(g, h)=\left(\begin{array}{cc}
\varphi\left(g h^{-1}\right) & \varphi\left(g \tau h^{-1}\right) \\
\varphi\left(g \tau h^{-1}\right) & \varphi\left(g h^{-1}\right)
\end{array}\right),
$$

and the function $\varphi$ on $G_{\tau}$ is positive definite if and only if $Q$ is positive definite.

Proof. That $Q$ is positive definite is equivalent to the exists of a Hilbert space $\mathcal{H}$ and a map

$$
\ell: G \rightarrow B(\mathcal{H}, V \oplus V) \cong B(\mathcal{H}, V)^{\oplus 2} \quad \text { with } \quad Q(x, y)=\ell(x) \ell(y)^{*} \quad \text { for } \quad x, y \in G
$$

(cf. [Ne00, Thm. I.1.4]). If $\ell$ is such a map, then it can be written as $\ell(x)=\left(\ell_{1}(x), \ell_{2}(x)\right)$ with $\ell_{j}(x) \in$ $B(\mathcal{H}, V)$. We thus obtain

$$
Q(x, y)=\ell(x) \ell(y)^{*}=\left(\begin{array}{ll}
\ell_{1}(x) \ell_{1}(y)^{*} & \ell_{1}(x) \ell_{2}(y)^{*} \\
\ell_{2}(x) \ell_{1}(y)^{*} & \ell_{2}(x) \ell_{2}(y)^{*}
\end{array}\right)
$$

and thus

$$
\ell_{1}(x) \ell_{1}(y)^{*}=\ell_{2}(x) \ell_{2}(y)^{*} \quad \text { and } \quad \ell_{1}(x) \ell_{2}(y)^{*}=\ell_{2}(x) \ell_{1}(y)^{*}
$$

Therefore

$$
j: G_{\tau} \rightarrow B(\mathcal{H}, V), \quad j(x, \mathbf{1}):=\ell_{1}(x), \quad j(x, \tau):=\ell_{2}(x)
$$

satisfies

$$
j(x, \mathbf{1}) j(y, \mathbf{1})^{*}=\ell_{1}(x) \ell_{1}(y)^{*}=\varphi\left(x y^{-1}\right), \quad j(x, \tau) j(y, \tau)^{*}=\ell_{2}(x) \ell_{2}(y)^{*}=\varphi\left(x y^{-1}\right)
$$

and

$$
j(x, \mathbf{1}) j(y, \tau)^{*}=\ell_{1}(x) \ell_{2}(y)^{*}=\varphi\left(x \tau y^{-1}\right), \quad j(x, \tau) j(y, \mathbf{1})^{*}=\ell_{2}(x) \ell_{1}(y)^{*}=\varphi\left(x y^{-1}\right) .
$$

We therefore have $\varphi\left(x y^{-1}\right)=j(x) j(y)^{*}$ for $x, y \in G_{\tau}$, and thus $\varphi$ is positive definite.

If, conversely, $\varphi$ is positive definite and $j: G_{\tau} \rightarrow B(\mathcal{H}, V)$ is such that $\varphi\left(x^{-1} y\right)=j(x) j(y)^{*}$ for $x, y \in G_{\tau}$, then $\ell(x):=(j(x, \mathbf{1}), j(x, \tau)) \in B(\mathcal{H}, V \oplus V)$ defines a map with $Q(x, y)=\ell(x) \ell(y)^{*}$ for $x, y \in G$.

Proposition 3.9. (GNS construction for reflection positive functions) Let $V$ be a real vector space, let $(U, \mathcal{E})$ be a unitary representation of $G_{\tau}$ and put $\theta:=U_{\tau}$. Then the following assertions hold: 
(i) If $(U, \mathcal{H}, j, V)$ is reflection positive w.r.t. $G_{+}$, then

$$
\varphi(g)(v, w):=\left\langle j(v), U_{g} j(w)\right\rangle, \quad g \in G_{\tau}, v, w \in V,
$$

is a reflection positive $\operatorname{Bil}(V)$-valued function.

(ii) If $\varphi: G_{\tau} \rightarrow \operatorname{Bil}(V)$ is a reflection positive function w.r.t. $G_{+}$, then the corresponding GNS representation $\left(U^{\varphi}, \mathcal{H}_{\varphi}, j, V\right)$ is a reflection positive representation, where $\mathcal{H}_{\varphi} \subseteq \mathbb{C}^{G_{\tau} \times V}$ is the Hilbert subspace with reproducing kernel $K((x, v),(y, w)):=\varphi\left(x y^{-1}\right)(v, w)$ on which $G_{\tau}$ acts by

$$
\left(U_{g}^{\varphi} f\right)(x, v):=f(x g, v) .
$$

Proof. (i) For $s, t \in G_{+}$, we have

$$
\varphi\left(s \tau t^{-1}\right)(v, w)=\left\langle j(v), U_{s \tau t^{-1}} j(w)\right\rangle=\left\langle U_{s^{-1}} j(v), U_{\tau} U_{t^{-1}} j(w)\right\rangle=\left\langle\theta U_{s^{-1}} j(v), U_{t^{-1}} j(w)\right\rangle,
$$

so that the kernel $\left(\varphi\left(s \tau t^{-1}\right)\right)_{s, t \in G_{+}}$is positive definite.

(ii) Recall the relation $\varphi(g)(v, w)=\left\langle j(v), U_{g} j(w)\right\rangle$ for $g \in G, v, w \in V$ from Proposition A.4 Moreover, $(\theta f)(x, v)=f(x \tau, v)$, and

$$
\left\langle\theta U_{s^{-1}}^{\varphi} j(v), U_{t^{-1}}^{\varphi} j(w)\right\rangle=\left\langle j(v), U_{s \tau t^{-1}}^{\varphi} j(w)\right\rangle=\varphi\left(s \tau t^{-1}\right)(v, w),
$$

so that the positive definiteness of the kernel $\left(\varphi\left(s \tau t^{-1}\right)\right)_{s, t \in G_{+}}$implies that we obtain with $\mathcal{E}=\mathcal{H}_{\varphi}$ and $\mathcal{E}_{+}:=\overline{\operatorname{span}\left(U_{G_{+}}^{\varphi}\right)^{-1} j(V)}$ a reflection positive Hilbert space $\left(\mathcal{E}, \mathcal{E}_{+}, \theta\right)$.

\subsection{Reflection positivity for the trivial group}

In this short section we discuss the case of the 2-element group $T=\{\mathbf{1}, \tau\}$ in some detail. It corresponds to $G_{\tau}$ where $G=\{\mathbf{1}\}$ is trivial, but it already demonstrates how the intricate structure of a reflection positive Hilbert space $\left(\mathcal{E}, \mathcal{E}_{+}, \theta\right)$ can be encoded in terms of positive definite functions on $T$.

A unitary representation $(U, \mathcal{E})$ of $T$ is nothing but the specification of a unitary operator $\theta=U_{\tau}$ on $\mathcal{E}$. We write $\mathcal{E}=\mathcal{E}^{1} \oplus \mathcal{E}^{-1}$ for the eigenspace decomposition of $\mathcal{E}$ under $\theta$ and $p^{ \pm 1}: \mathcal{E} \rightarrow \mathcal{E}^{ \pm 1}$ for the orthogonal projections.

Suppose, in addition, that $V$ be a real or complex Hilbert space and that $j: V \rightarrow \mathcal{E}$ is a continuous linear map whose range generates $\mathcal{E}$ under the representation $U$, i.e., the projections $p^{ \pm 1}(j(V)) \subseteq \mathcal{E}^{ \pm 1}$ are dense subspaces. In view of the GNS construction, the data $(\mathcal{E}, U, j, V)$ is encoded in the operator-valued positive definite function

$$
\varphi: T \rightarrow B(V), \quad \varphi(g)=j^{*} U_{g} j .
$$

For a function $\varphi: T \rightarrow B(V)$, let $A:=\varphi(\mathbf{1})$ and $B:=\varphi(\tau)$. Then $\varphi$ is positive definite if and only if $A=A^{*} \geq 0, B=B^{*}$, and the operator matrix

$$
\left(\begin{array}{ll}
\varphi(\mathbf{1}) & \varphi(\tau) \\
\varphi(\tau) & \varphi(\mathbf{1})
\end{array}\right)=\left(\begin{array}{ll}
A & B \\
B & A
\end{array}\right) \in M_{2}(B(V)) \cong B(V \oplus V)
$$

defines a positive operator (Lemma 3.8 and [Ne00, Rem. I.1.3]). This is equivalent to

$$
|\langle B v, w\rangle|^{2} \leq\langle A v, v\rangle\langle A w, w\rangle \quad \text { for } \quad v, w \in V
$$


(cf. Corollary A.9). Note that (13) holds if $A=\mathbf{1}$ and $\|B\| \leq 1$. If, more generally, $A$ is invertible, then (13) is equivalent to $\left\|A^{-1 / 2} B A^{-1 / 2}\right\| \leq \mathbf{1}$. Here $A=j^{*} j$ basically encodes how $V$ is mapped into $\mathcal{E}$ and $B$ encodes the unitary involution $\theta$.

The function $\varphi$ is reflection positive w.r.t. $G_{+}=\{\mathbf{1}\}$ if and only if $B=\varphi(\tau) \geq 0$, which means that $j(V)$ is $\theta$-positive. In this sense reflection positive functions on $T$ encode reflection positive Hilbert spaces $\left(\mathcal{E}, \mathcal{E}_{+}, \theta\right)$ by $\theta=U_{\tau}$ and $\mathcal{E}_{+}:=\overline{j(V)}$. A pair $(A, B)$ of hermitian operators on $V$ corresponds to a reflection positive function $\varphi: T \rightarrow B(V)$ if and only if $0 \leq B \leq A$. By the Cauchy-Schwarz inequality, this is equivalent to (13) if $A$ and $B$ are positive operators. This shows that

$$
\varphi=\varphi_{0}+\varphi_{1} \quad \text { with } \quad \varphi_{0}(\mathbf{1})=A-B, \quad \varphi_{0}(\tau)=0 \quad \text { and } \quad \varphi_{1}(\mathbf{1})=\varphi_{1}(\tau)=B,
$$

where both functions $\varphi_{0}$ and $\varphi_{1}$ are reflection positive. The function $\varphi_{0}$ corresponds to the case where $\mathcal{E}_{+} \perp \theta \mathcal{E}_{+}$, so that $\widehat{\mathcal{E}}=\{0\}$, and the constant function $\varphi_{1}$ corresponds to the trivial representation of $T$, hence to $\theta=\mathbf{1}$, which means that $q: \mathcal{E}_{+} \rightarrow \widehat{\mathcal{E}}$ is isometric.

Replacing $V$ by $\mathcal{E}_{+}$, we see that reflection positive functions $\varphi: T \rightarrow B\left(\mathcal{E}_{+}\right)$with $\varphi(\mathbf{1})=\mathbf{1}$ encode reflection positive Hilbert spaces $\left(\mathcal{E}, \mathcal{E}_{+}, \theta\right)$ for which $p^{ \pm 1}\left(\mathcal{E}_{+}\right)$is dense in $\mathcal{E}^{ \pm 1}$. By (13), these configurations are parametrized by the hermitian contractions $B=\varphi(\tau)$ on $\mathcal{E}_{+}$. For $v, w \in \mathcal{E}_{+}$, we then have

$$
\langle v, \theta w\rangle=\langle v, B w\rangle \text {. }
$$

Therefore the 1-eigenspace $\operatorname{ker}(B-\mathbf{1})$ corresponds to the maximal subspace in $\mathcal{E}_{+}$on which the map $q: \mathcal{E}_{+} \rightarrow \widehat{\mathcal{E}}$ is isometric. We also observe that $\operatorname{ker} B=\operatorname{ker} q$. In this sense the operator $B$ describes how $\widehat{\mathcal{E}}$ is obtained from the Hilbert space $\mathcal{E}_{+}$.

Remark 3.10. Suppose that $\theta$ is a unitary involution on $\mathcal{E}$ with the eigenspaces $\mathcal{E}^{ \pm 1}$. If $\mathcal{K} \subseteq \mathcal{E}$ is a $\theta$-positive subspace, then clearly $\mathcal{K} \cap \mathcal{E}^{-1}=\{0\}$ and this implies that $\mathcal{K}$ is the graph $\Gamma(Z)$ of the operator

$$
Z: \mathcal{D}(Z):=\left\{v_{+} \in \mathcal{E}^{1}:\left(\exists v_{-} \in \mathcal{E}^{-1}\right)\left(v_{+}, v_{-}\right) \in \mathcal{K}\right\} \rightarrow \mathcal{E}^{-1}, \quad v_{+} \mapsto v_{-}
$$

That $\Gamma(Z)$ is a $\theta$-positive subspace is equivalent to $\|Z\| \leq 1$. Therefore the closedness of $\mathcal{K}$ shows that $\mathcal{D}(Z)$ is a closed subspace of $\mathcal{E}^{1}$ (cf. Jo02, Lemma 5.1]). If $p^{1}(\mathcal{K})=\mathcal{D}(Z)$ is dense in $\widehat{\mathcal{E}}$, the closedness of $\mathcal{D}(Z)$ implies that $Z \in B\left(\mathcal{E}^{1}, \mathcal{E}^{-1}\right)$. The density of $p^{-1}(\mathcal{K})=Z\left(\mathcal{E}^{1}\right)$ is equivalent to $Z$ having dense range.

¿From this perspective, we can also generate the configuration $\left(\mathcal{E}, \mathcal{E}_{+}, \theta\right)$ in terms of $\mathcal{E}^{1}$. Then $j(v)=$ $(v, Z v) \in \mathcal{E}^{1} \oplus \mathcal{E}^{-1}$ defines a linear map $j: \mathcal{E}^{1} \rightarrow \mathcal{E}$ whose range is $\mathcal{K}$. The corresponding $B\left(\mathcal{E}^{1}\right)$-valued positive definite function on $T$ is given by

$$
\psi(\mathbf{1})=j^{*} j=\mathbf{1}+Z^{*} Z \quad \text { and } \quad \psi(\tau)=j^{*} \theta j=\mathbf{1}-Z^{*} Z .
$$

The polar decomposition of $j: \mathcal{E}^{1} \rightarrow \mathcal{K}$ takes the form

$$
j=U \sqrt{j^{*} j}=U \sqrt{\mathbf{1 + Z} Z}
$$

where $U: \mathcal{E}^{1} \rightarrow \mathcal{K}$ is unitary. Therefore the corresponding $B(\mathcal{K})$-valued positive definite function on $T$ is given by

$$
\varphi(\mathbf{1})=\mathbf{1} \quad \text { and } \quad \varphi(\tau)=U \frac{\mathbf{1}-Z^{*} Z}{\mathbf{1}+Z^{*} Z} U^{-1}
$$

because $j^{*} \varphi(\tau) j=j^{*} \theta j=\mathbf{1}-Z^{*} Z$ implies

$$
\varphi(\tau)=\left(j^{*}\right)^{-1}\left(\mathbf{1}-Z^{*} Z\right) j^{-1}=U\left(\mathbf{1}+Z^{*} Z\right)^{-1 / 2}\left(\mathbf{1}-Z^{*} Z\right)\left(\mathbf{1}+Z^{*} Z\right)^{-1 / 2} U^{-1}=U \frac{\mathbf{1}-Z^{*} Z}{\mathbf{1}+Z^{*} Z} U^{-1}
$$


Relating this to the preceding discussion, we see that $U$ ker $Z \subseteq \mathcal{E}_{+}$is the maximal subspace on which $q$ is isometric and

$$
U\left\{v \in \mathcal{E}^{1}:\|Z v\|=\|v\|\right\}=U \operatorname{ker}\left(\mathbf{1}-Z^{*} Z\right)=\operatorname{ker} q .
$$

In particular, $q$ is injective if and only if $Z$ is a strict contraction.

\subsection{Reflection positivity for the 2-element group}

In this subsection we take a closer look at the 2-element group $G=\{\mathbf{1}, \sigma\}$ because it nicely illustrates that, if a reflection positive representation decomposes into two subrepresentations, then the summands need not be reflection positive (see also [NÓ14a]). On the level of positive definite functions, this is reflected in the fact that the cone of reflection positive functions does not adapt to the decomposition into even and odd functions.

We consider the 2-element group $G:=\{\mathbf{1}, \sigma\}$, which leads to the Klein-4-group

$$
G_{\tau}:=G \rtimes\{\mathbf{1}, \tau\} \cong \mathbb{Z} / 2 \mathbb{Z} \times \mathbb{Z} / 2 \mathbb{Z} .
$$

We consider reflection positivity with respect to the subset $G_{+}:=\{\mathbf{1}\}$.

Any unitary representation $(U, \mathcal{E})$ of $G_{\tau}$ decomposes into four eigenspaces

$$
\mathcal{E}=\mathcal{E}^{1,1} \oplus \mathcal{E}^{-1,1} \oplus \mathcal{E}^{1,-1} \oplus \mathcal{E}^{-1,-1}, \quad \mathcal{E}^{\varepsilon_{1}, \varepsilon_{2}}=\left\{v \in \mathcal{E}: U_{\sigma} v=\varepsilon_{1} v, U_{\tau} v=\varepsilon_{2} v\right\},
$$

and for $\theta:=U_{\tau}$, the subspace $\mathcal{E}^{\theta}=\mathcal{E}^{1,1} \oplus \mathcal{E}^{-1,1}$ is $U_{\sigma}$-invariant. For $v=(a, b, c, d)$, we then have

$$
U_{\tau} v=(a, b,-c,-d) \quad \text { and } \quad U_{\sigma} v=(a,-b, c,-d) .
$$

Assume that $\mathcal{E}_{+}=\mathbb{C} v$ for a single vector $v$. Then reflection positivity corresponds to

$$
\langle v, \theta v\rangle=|a|^{2}+|b|^{2}-|c|^{2}-|d|^{2} \geq 0 .
$$

With respect to $U_{\sigma}$, we have

$$
v=v_{1}+v_{-1}=(a, 0, c, 0)+(0, b, 0, d)
$$

and

$$
\left\langle U_{\sigma} v, \theta U_{\sigma} v\right\rangle=\langle v, \theta v\rangle \geq 0 \quad \text { and } \quad\left\langle U_{\sigma} v, \theta v\right\rangle=|a|^{2}-|b|^{2}-|c|^{2}+|d|^{2} .
$$

Therefore the subspace $\mathbb{C} v+\mathbb{C} U_{\sigma} v$ is $\theta$-positive if and only if

$$
\pm\left(|a|^{2}-|b|^{2}-|c|^{2}+|d|^{2}\right) \leq|a|^{2}+|b|^{2}-|c|^{2}-|d|^{2},
$$

which is equivalent to

$$
|d| \leq|b| \quad \text { and } \quad|c| \leq|a| .
$$

Clearly, these two conditions are strictly stronger than the $\theta$-positivity of $\mathbb{C} v$.

For the corresponding positive definite function $f(g)=\left\langle v, U_{g} v\right\rangle$ we have

$$
\begin{aligned}
f(\mathbf{1}) & =|a|^{2}+|b|^{2}+|c|^{2}+|d|^{2}, & f(\tau) & =|a|^{2}+|b|^{2}-|c|^{2}-|d|^{2}, \\
f(\sigma) & =|a|^{2}-|b|^{2}+|c|^{2}-|d|^{2}, & f(\sigma \tau) & =|a|^{2}-|b|^{2}-|c|^{2}+|d|^{2} .
\end{aligned}
$$


Decomposing $f=f_{1}+f_{-1}$ with respect to the left action of $\sigma$, we obtain

$$
f_{1}(\mathbf{1})=f_{1}(\sigma)=|a|^{2}+|c|^{2}, \quad f_{1}(\tau)=f_{1}(\sigma \tau)=|a|^{2}-|c|^{2}
$$

and

$$
f_{-1}(\mathbf{1})=-f_{1}(\sigma)=|b|^{2}+|d|^{2}, \quad f_{-1}(\tau)=-f_{-1}(\sigma \tau)=|b|^{2}-|d|^{2} .
$$

Both functions $f_{ \pm 1}(g)=\left\langle v_{ \pm 1}, U_{g} v_{ \pm 1}\right\rangle$ are positive definite, but they are reflection positive if and only if $|c| \leq|a|$ and $|d| \leq|b|$.

Note that, even for $U_{\sigma}=\mathbf{1}$ and $U_{\sigma}=-\mathbf{1}$, there exist non-trivial reflection positive representations with $\langle v, \theta v\rangle>0$.

\section{Reflection positive functions and KMS conditions}

In this section we build the bridge from positive definite functions $\psi: \mathbb{R} \rightarrow \operatorname{Bil}(V)$ satisfying the KMS condition for $\beta>0$ to reflection positive functions on the group $\mathbb{T}_{2 \beta, \tau} \cong \mathrm{O}_{2}(\mathbb{R})$. We have already seen in Lemma 2.2 that analytic continuation leads to a $2 \beta$-periodic function $\varphi: \mathbb{R} \rightarrow \operatorname{Bil}(V)$ satisfying $\varphi(t+\beta)=\overline{\varphi(t)}$ for $t \in \mathbb{R}$ and $\varphi(t)=\psi(i t)$ for $0 \leq t \leq \beta$. In this section we show the existence of a positive definite function $f: \mathbb{R}_{\tau} \rightarrow \operatorname{Bil}(V)$ with $f(t, \tau)=\varphi(t)$ for $t \in \mathbb{R}$. By construction, $f$ is then reflection positive with respect to the interval $[0, \beta / 2]=G_{+} \subseteq G=\mathbb{R}$ in the sense of Definition 3.4.

Since we can build on Theorem 2.6, our first goal is to express, for a standard real subspace $V \subseteq \mathcal{H}$, the $\operatorname{Bil}(V)$-valued function

$$
\varphi:[0, \beta] \rightarrow \operatorname{Bil}(V), \quad \varphi(t)(v, w):=\psi(i t)(v, w)=\left\langle\Delta^{t / 2 \beta} v, \Delta^{t / 2 \beta} w\right\rangle \quad \text { for } \quad v, w \in V, 0 \leq t \leq \beta
$$

from (8) in the proof of Theorem[2.6] as a $B\left(V_{\mathbb{C}}\right)$-valued function. To this end, we shall need the description of $V$ in terms of a skew-symmetric strict contraction $C$ on $V$ (Lemma B.9), and this leads to a quite explicit description of $\varphi$ that we then use to prove our main theorem.

\subsection{From form-valued to operator-valued functions}

In the following it will be more convenient to work with operator-valued functions instead of form-valued ones. The translation is achieved by the following lemma. For its formulation, we recall the polar decomposition of bounded skew-symmetric operators on real Hilbert spaces.

Remark 4.1. (Polar decomposition of skew-symmetric operators) Let $D^{\top}=-D$ be an injective skewsymmetric operator on the real Hilbert space $V$ and let $D=I|D|$ be its polar decomposition. Then $\operatorname{im}(D)$ is dense because $D$ is injective, and therefore $I$ defines an isometry $V \rightarrow V$. From

$$
I|D|=D=-D^{\top}=-|D| I^{-1}=-I^{-1}\left(I|D| I^{-1}\right)
$$

it follows that $I^{2}=-\mathbf{1}$, i.e., that $I$ is a complex structure and that $|D|$ commutes with $I$.

Lemma 4.2. Let $V \subseteq \mathcal{H}$ be a standard real subspace with modular objects $(\Delta, J)$, let $\widehat{C}:=i \frac{\Delta-\mathbf{1}}{\Delta+\mathbf{1}}$, and let $C:=\left.\widehat{C}\right|_{V} \in B(V)$ be the skew-symmetric strict contraction from Lemma B.9. We assume that $\operatorname{ker} C=\{0\}$, so that the polar decomposition $C=I|C|$ defines a complex structure $I$ on $V$. Consider the skew-symmetric operator

$$
D:=\log \left(\frac{\mathbf{1}-|C|}{\mathbf{1}+|C|}\right) I \text {. }
$$


Then the function $\varphi(t)(v, w)=\left\langle\Delta^{t / 2} v, \Delta^{t / 2} w\right\rangle$ from (14) has the form

$$
\varphi(t)(v, w)=\langle v, \widetilde{\varphi}(t) w\rangle_{V_{\mathbb{C}}} \quad \text { for } \quad t \in[0,1], v, w \in V_{\mathbb{C}},
$$

where the function $\widetilde{\varphi}:[0,1] \rightarrow B\left(V_{\mathbb{C}}\right)$ is given by

$$
\widetilde{\varphi}(t)=(\mathbf{1}+i C)^{1-t}(\mathbf{1}-i C)^{t}=\frac{e^{-t|D|}+e^{-(1-t)|D|}}{\mathbf{1}+e^{-|D|}}+i I \frac{e^{-t|D|}-e^{-(1-t)|D|}}{\mathbf{1}+e^{-|D|}} .
$$

Note that $\widetilde{\varphi}(0)=\mathbf{1}+i C \neq \mathbf{1}$ if $C \neq 0$.

Proof. Since $C$ is a skew-symmetric contraction on $V$, the operators $\mathbf{1} \pm i C$ on $V_{\mathbb{C}}$ are symmetric, so that we obtain a function

$$
\widetilde{\varphi}:[0,1] \rightarrow B\left(V_{\mathbb{C}}\right), \quad \widetilde{\varphi}(t):=(\mathbf{1}+i C)^{1-t}(\mathbf{1}-i C)^{t}, \quad 0 \leq t \leq 1 .
$$

Therefore both sides of (15) are defined, and we have to show that

$$
\langle v, \widetilde{\varphi}(t) w\rangle_{V_{\mathbb{C}}}=\left\langle\Delta^{t / 2} v, \Delta^{t / 2} w\right\rangle \quad \text { for } \quad v, w \in V_{\mathbb{C}} .
$$

For the skew-hermitian contraction $\widehat{C}$ on $\mathcal{H}$, we likewise obtain bounded operators

$$
\widehat{\varphi}(t):=(\mathbf{1}+i \widehat{C})^{1-t}(\mathbf{1}-i \widehat{C})^{t}, \quad 0 \leq t \leq 1,
$$

and the continuity of the inclusion $V_{\mathbb{C}} \hookrightarrow \mathcal{H}$ implies that

$$
\left.\widehat{\varphi}(t)\right|_{V_{\mathbb{C}}}=\widetilde{\varphi}(t): V_{\mathbb{C}} \rightarrow V_{\mathbb{C}} .
$$

¿From the relation $\Delta=\frac{1-i \widehat{C}}{1+i \widehat{C}}$ we further obtain the identity

$$
\widehat{\varphi}(t)=(\mathbf{1}+i \widehat{C}) \Delta^{t}
$$

of selfadjoint operators on $\mathcal{H}$. Let $V_{\mathbb{C}}^{\prime}$ denote the domain of the (possibly) unbounded selfadjoint operator $\frac{1-i C}{1+i C}$ on $V_{\mathbb{C}}$. Then $V_{\mathbb{C}}^{\prime}$ is a dense subspace which is contained in the domain of $\left(\frac{1-i C}{1+i C}\right)^{t}$ for $0 \leq t \leq 1$. For $w \in V_{\mathbb{C}}^{\prime}$ we have

$$
\widetilde{\varphi}(t) w=(\mathbf{1}+i C)\left(\frac{\mathbf{1}-i C}{\mathbf{1}+i C}\right)^{t} w \quad \text { for } \quad 0 \leq t \leq 1 .
$$

For $v \in V_{\mathbb{C}}$ and $\widetilde{w}:=\left(\frac{1-i C}{1+i C}\right)^{t} w$ we now obtain with (39) from Lemma B.9 the relation

$$
\begin{aligned}
\langle v, \widetilde{\varphi}(t) w\rangle_{V_{\mathbb{C}}} & =\langle v,(\mathbf{1}+i C) \widetilde{w}\rangle_{V_{\mathbb{C}}}=\langle v, \widetilde{w}\rangle_{\mathcal{H}}=\left\langle v,\left(\frac{\mathbf{1}-i C}{\mathbf{1}+i C}\right)^{t} w\right\rangle_{\mathcal{H}} \\
& =\left\langle v,\left(\frac{\mathbf{1}-i \widehat{C}}{\mathbf{1}+i \widehat{C}}\right)^{t} w\right\rangle_{\mathcal{H}}=\left\langle v, \Delta^{t} w\right\rangle_{\mathcal{H}}=\left\langle\Delta^{t / 2} v, \Delta^{t / 2} w\right\rangle_{\mathcal{H}} .
\end{aligned}
$$

Since both sides of (16) define continuous hermitian forms on $V_{\mathbb{C}}$ and the preceding calculation shows that equality holds on a dense subspace, we obtain (16) for all $v, w \in V_{\mathbb{C}}$. 
Next we observe that the polar decomposition of $D$ is given by

$$
D=-I|D| \quad \text { and } \quad|D|=\log \left(\frac{\mathbf{1}+|C|}{\mathbf{1}-|C|}\right)
$$

The operator $|D|$ satisfies

$$
e^{\mp|D|}=\frac{\mathbf{1} \mp|C|}{\mathbf{1} \pm|C|} \quad \text { and } \quad 1+e^{\mp|D|}=\frac{2}{1 \pm|C|} .
$$

Since $i I$ is an involution with the two eigenvalues \pm 1 , comparing the action on both eigenspaces shows that, for $0 \leq t \leq 1$, we have

$$
\left(\frac{\mathbf{1}-i C}{\mathbf{1}+i C}\right)^{t}=\left(\frac{\mathbf{1}-i I|C|}{\mathbf{1}+i I|C|}\right)^{t}=e^{-t|D| i I} .
$$

The assertion of the lemma now follows from

$$
\begin{aligned}
\widetilde{\varphi}(t) & =(\mathbf{1}+i C)\left(\frac{\mathbf{1}-i C}{\mathbf{1}+i C}\right)^{t}=(\mathbf{1}+i I|C|) e^{-t|D| i I}=(\mathbf{1}+i I|C|)\left(e^{t|D|} \frac{\mathbf{1}-i I}{2}+e^{-t|D|} \frac{\mathbf{1}+i I}{2}\right) \\
& =e^{-t|D|}(\mathbf{1}+|C|) \frac{\mathbf{1}+i I}{2}+e^{t|D|}(\mathbf{1}-|C|) \frac{\mathbf{1}-i I}{2}=(\mathbf{1}+|C|)\left(e^{-t|D|} \frac{\mathbf{1}+i I}{2}+e^{-(1-t)|D|} \frac{\mathbf{1}-i I}{2}\right) \\
& =\left(\mathbf{1}+e^{-|D|}\right)^{-1}\left(e^{-t|D|}(\mathbf{1}+i I)+e^{-(1-t)|D|}(\mathbf{1}-i I)\right)=\frac{e^{-t|D|}+e^{-(1-t)|D|}}{\mathbf{1}+e^{-|D|}}+i I \frac{e^{-t|D|}-e^{-(1-t)|D|}}{\mathbf{1}+e^{-|D|}} .
\end{aligned}
$$

Remark 4.3. (a) Since $C$ is a strict contraction on $V, \mathbf{1}+i C$ is injective on $V_{\mathbb{C}}$, so that

$$
\widetilde{H}:=\log \left(\frac{\mathbf{1}+i C}{\mathbf{1}-i C}\right)=\log \left(\frac{\mathbf{1}+i I|C|}{\mathbf{1}-i I|C|}\right)=i I|D|=-i D
$$

also defines a selfadjoint operator on the complex Hilbert space space $V_{\mathbb{C}}$.

Next we observe that $\widetilde{H}$ is a restriction of $L:=\log \left(\frac{1+i \widehat{C}}{1-i C}\right)=-\log \Delta$, the infinitesimal generator of the one-parameter group $U_{t}=\Delta^{-i t}$. For the orthogonal one-parameter group $U_{t}^{V}:=\left.U_{t}\right|_{V} \in \mathrm{O}(V)$, it follows that its infinitesimal generator is a skew-adjoint extension of the skew-adjoint operator $D$ on $V$, hence coincides with $D$. We therefore have

$$
e^{t D}=\left.\Delta^{-i t}\right|_{V} \quad \text { for } \quad t \in \mathbb{R} .
$$

This provides an alternative characterization of the operator $D$ in Lemma 4.2

(b) Let $(V,(\cdot, \cdot))$ be a real Hilbert space and $\left(U_{t}\right)_{t \in \mathbb{R}}$ be an orthogonal strongly continuous oneparameter group with skew-symmetric infinitesimal generator $D$, i.e., $U_{t}=e^{t D}$ for $t \in \mathbb{R}$. Let us assume that $\operatorname{ker} D=\{0\}$, resp., that the subspace $V^{U}$ of $U$-fixed points in $V$ is trivial. Then the polar decomposition $D=I|D|$ can be used to define a skew-symmetric contraction

$$
C:=(-I) \frac{1-e^{-|D|}}{1+e^{-|D|}} \quad \text { with } \quad|C|=\frac{1-e^{-|D|}}{1+e^{-|D|}}
$$

Then the hermitian form

$$
h(v, w):=(v, w)+i(v, C w)
$$


defines a positive definite kernel on $V$ (Lemma A.10). Let $\mathcal{H}$ denote the corresponding reproducing kernel space and let $j: V \rightarrow \mathcal{H}$ be the natural map. By construction, $|C|$ has no fixed points, so that $\mathbf{1}+C^{2}$ is injective, and therefore Lemma A.10(iii) implies that the complex linear extension $j_{\mathbb{C}}: V_{\mathbb{C}} \rightarrow \mathcal{H}$ is injective. As the real part of $h$ is the original scalar product on $V$, the inclusion $V \hookrightarrow \mathcal{H}$ is isometric, so that $V \cong j(V)$ is a standard real subspace of $\mathcal{H}$. Since $h$ is $U$-invariant, it defines a unitary one-parameter group $\widehat{U}$ on $\mathcal{H}$. Finally (18) implies that $\widehat{U}_{t}=\Delta^{-i t}$ for $t \in \mathbb{R}$ and the modular operator $\Delta$ corresponding to $j(V)$. This shows that every orthogonal one-parameter group on a real Hilbert space is of the form (18) for a naturally defined embedding $V \hookrightarrow \mathcal{H}$ as a standard real subspace.

Before we turn to the associated reflection positive functions, we need the following technical lemma on Fourier expansions of certain operator-valued functions. In [CMV01 this is called the Matsubara

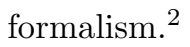

Lemma 4.4. Let $B \geq 0$ be a selfadjoint operator on the complex Hilbert space $\mathcal{H}$ and let $\beta>0$. We consider the operator-valued functions $u_{B}^{ \pm}: \mathbb{R} \rightarrow B(\mathcal{H})$ satisfying

$$
u_{B}^{ \pm}(t+\beta)= \pm u_{B}^{ \pm}(t) \quad \text { and } \quad u_{B}^{ \pm}(t)=\frac{e^{-t B} \pm e^{-(\beta-t) B}}{1+e^{-\beta B}} \quad \text { for } \quad 0 \leq t \leq \beta .
$$

Then $u_{B}^{ \pm}$are weakly continuous symmetric $2 \beta$-periodic with the Fourier expansions

$$
u_{B}^{+}(t)=\sum_{n \in \mathbb{Z}} c_{2 n}^{B} e^{2 n \pi i t / \beta} \quad \text { and } \quad u_{B}^{-}(t)=\sum_{n \in \mathbb{Z}} c_{2 n+1}^{B} e^{(2 n+1) \pi i t / \beta}
$$

with

$$
c_{n}^{B}=c_{-n}^{B}=\frac{\left(\mathbf{1}-(-1)^{n} e^{-\beta B}\right)}{\mathbf{1}+e^{-\beta B}} \cdot \frac{2 \beta B}{(\beta B)^{2}+(n \pi)^{2}} \quad \text { for } \quad n \in \mathbb{Z}
$$

Proof. (a) Every $2 \beta$-periodic continuous function $\xi: \mathbb{R} \rightarrow \mathbb{C}$ has a Fourier expansion

$$
\xi(t)=\sum_{n \in \mathbb{Z}} c_{n} e^{\pi i n t / \beta} \quad \text { with } \quad c_{n}=\frac{1}{2 \beta} \int_{0}^{2 \beta} \xi(t) e^{-\pi i n t / \beta} d t .
$$

For the $\beta$-periodic function with $u^{+}(t)=u_{\lambda}^{+}(t):=\frac{e^{-t \lambda}+e^{-(\beta-t) \lambda}}{1+e^{-\beta \lambda}}$ for $0 \leq t \leq \beta$ we have $u^{+}(t+\beta)=u^{+}(t)$, so that only even terms appear:

$$
u^{+}(t)=\sum_{n \in \mathbb{Z}} c_{2 n} e^{\pi i 2 n t / \beta}, \quad c_{2 n}=\frac{1-e^{-\beta \lambda}}{1+e^{-\beta \lambda}} \frac{2 \beta \lambda}{(\beta \lambda)^{2}+(2 \pi n)^{2}} .
$$

To obtain this formula, we first calculate

$$
a_{\lambda, n}:=\frac{1}{\beta} \int_{0}^{\beta} e^{-t \lambda} e^{-\pi i n t / \beta} d t=\int_{0}^{1} e^{-(\beta \lambda+\pi i n) t} d t=\frac{1-e^{-(\beta \lambda+\pi i n)}}{\beta \lambda+\pi i 2 n}=\frac{1-(-1)^{n} e^{-\beta \lambda}}{\beta \lambda+\pi i 2 n} .
$$

${ }^{2}$ In view of [DG13 Def. 18.49], we have

$$
u_{B}^{+}(t)=G_{E, \beta}(t) \cdot \frac{2 B\left(\mathbf{1}-e^{-\beta B}\right)}{\mathbf{1}+e^{-\beta B}},
$$

where $G_{E, \beta}$ is the euclidean thermal Green's function associated to the positive operator $\varepsilon=B$. 
Therefore

$$
\begin{aligned}
\left(1+e^{-\lambda \beta}\right) c_{2 n} & =a_{\lambda, 2 n}+e^{-\beta \lambda} a_{-\lambda, 2 n}=\frac{1-e^{-\beta \lambda}}{\beta \lambda+2 n \pi i}+e^{-\beta \lambda} \frac{1-e^{\beta \lambda}}{-\beta \lambda+2 n \pi i} \\
& =\frac{1-e^{-\beta \lambda}}{\beta \lambda+2 n \pi i}+\frac{1-e^{-\beta \lambda}}{\beta \lambda-2 n \pi i}=\frac{\left(1-e^{-\beta \lambda}\right) 2 \beta \lambda}{(\beta \lambda)^{2}+(2 n \pi)^{2}}
\end{aligned}
$$

For the $2 \beta$-periodic function with $u^{-}(t)=u_{\lambda}^{-}(t):=\frac{e^{-t \lambda}-e^{-(\beta-t) \lambda}}{1+e^{-\beta \lambda}}$ for $0 \leq t \leq \beta$ and $u^{-}(t+\beta)=-u^{-}(t)$ only odd terms appear:

$$
u^{-}(t)=\sum_{n \in \mathbb{Z}} c_{2 n+1} e^{\pi i(2 n+1) t / \beta}, \quad c_{2 n+1}=\frac{2 \beta \lambda}{(\beta \lambda)^{2}+((2 n+1) \pi)^{2}} .
$$

This follows from

$$
\begin{aligned}
c_{2 n+1} & =\frac{a_{\lambda, 2 n+1}-e^{-\beta \lambda} a_{-\lambda, 2 n+1}}{1+e^{-\beta \lambda}}=\frac{1}{\beta \lambda+(2 n+1) \pi i}-\frac{e^{-\beta \lambda}\left(1+e^{\beta \lambda}\right)}{1+e^{-\beta \lambda}} \frac{1}{-\beta \lambda+(2 n+1) \pi i} \\
& =\frac{1}{\beta \lambda+(2 n+1) \pi i}+\frac{1}{\beta \lambda-(2 n+1) \pi i}=\frac{2 \beta \lambda}{(\beta \lambda)^{2}+((2 n+1) \pi)^{2}}
\end{aligned}
$$

Note that

$$
c_{n}=c_{-n}=\frac{1-(-1)^{n} e^{-\beta \lambda}}{1+e^{-\beta \lambda}} \frac{2 \beta \lambda}{(\beta \lambda)^{2}+(n \pi)^{2}} \quad \text { for } \quad n \in \mathbb{Z} .
$$

(b) If $P$ denotes the spectral measure of $B$, we have for $v \in \mathcal{H}$ the relation

$$
\langle v, B v\rangle=\int_{0}^{\infty} x d P^{v, v}(x) \quad \text { with } \quad P^{v, v}=\langle v, P(\cdot) v\rangle
$$

This leads for $0 \leq t \leq 2 \beta$ to

$$
\left\langle v, u_{B}^{ \pm}(t) v\right\rangle=\int_{0}^{\infty} u_{\lambda}^{ \pm}(t) d P^{v, v}(\lambda)
$$

For the operator-valued Fourier coefficients, we thus obtain

$$
\begin{aligned}
\left\langle v, c_{n}^{B} v\right\rangle & =\int_{\mathbb{R}} c_{n}(\lambda) d P^{v, v}(\lambda)=\int_{\mathbb{R}} \frac{1-(-1)^{n} e^{-\beta \lambda}}{1+e^{-\beta \lambda}} \frac{2 \beta \lambda}{(\beta \lambda)^{2}+(n \pi)^{2}} d P^{v, v}(\lambda) \\
& =\left\langle v, \frac{\left(\mathbf{1}-(-1)^{n} e^{-\beta B}\right)}{\mathbf{1}+e^{-\beta B}} \frac{2 \beta B}{(\beta B)^{2}+(n \pi)^{2}} v\right\rangle .
\end{aligned}
$$

This proves the assertion.

\subsection{Existence of reflection positive extensions}

We now come to one of our main result on reflection positive extensions. It shows that, for every positive definite function $\psi: \mathbb{R} \rightarrow \operatorname{Bil}(V)$ satisfying the $\beta$-KMS condition, there exists a reflection positive function $f: G_{\tau} \rightarrow B\left(V_{\mathbb{C}}\right)$ satisfying $\psi(i t)(v, w)=\langle v, f(i t, \tau) w\rangle$ for $v, w \in V, 0 \leq t \leq \beta$. Then the corresponding GNS representation $\left(U^{f}, \mathcal{H}_{f}\right)$ of the group $\left(\mathbb{T}_{2 \beta}\right)_{\tau} \cong \mathrm{O}_{2}(\mathbb{R})$ is a "euclidean realization" of the unitary oneparameter group $\left(\Delta^{-i t / \beta}\right)_{t \in \mathbb{R}}$ corresponding to $\psi$ in the sense that it is obtained by Osterwalder-Schrader quantization from $U^{f}$ (cf. NÓ14a]). The following theorem generalizes the results of NÓ15] dealing with the scalar-valued case. 
Theorem 4.5. (Reflection positive extensions) Let $V \subseteq \mathcal{H}$ be a standard real subspace and let $C=I|C|$ be the corresponding skew-symmetric strict contraction on $V$. We assume that $\operatorname{ker} C=\{0\}$, so that $I$ defines a complex structure on $V$. We define a weakly continuous function $\widetilde{\varphi}: \mathbb{R} \rightarrow B\left(V_{\mathbb{C}}\right)$ by

$$
\widetilde{\varphi}(t)=(\mathbf{1}+i C)^{1-t / \beta}(\mathbf{1}-i C)^{t / \beta} \quad \text { for } \quad 0 \leq t \leq \beta \quad \text { and } \quad \widetilde{\varphi}(t+\beta)=\overline{\widetilde{\varphi}(t)} \quad \text { for } \quad t \in \mathbb{R} .
$$

Write

$$
\widetilde{\varphi}(t)=u^{+}(t)+i I u^{-}(t) \quad \text { with } \quad u^{ \pm}(t) \in B(V), \quad u^{ \pm}(t+\beta)= \pm u^{ \pm}(t) .
$$

Then

$$
f: \mathbb{R}_{\tau} \rightarrow B\left(V_{\mathbb{C}}\right), \quad f\left(t, \tau^{\varepsilon}\right):=u^{+}(t)+(i I)^{\varepsilon} u^{-}(t), \quad t \in \mathbb{R}, \varepsilon \in\{0,1\},
$$

is a weak-operator continuous positive definite function satisfying $f(t, \tau)=\widetilde{\varphi}(t)$. It is reflection positive with respect to the subset $[0, \beta / 2] \subseteq \mathbb{R}$ in the sense that the kernel

$$
f((t, \tau)(-s, \mathbf{1}))=f(t+s, \tau), \quad 0 \leq s, t \leq \beta / 2
$$

is positive definite.

Proof. We may w.l.o.g. assume that $\beta=1$. Recall the operator $D$ from Lemma 4.2. With this lemma, we write

$$
\widetilde{\varphi}(t)=\left(\mathbf{1}+e^{-|D|}\right)^{-1}\left(e^{-t|D|}+e^{-(1-t)|D|}+i I\left(e^{-t|D|}-e^{-(1-t)|D|}\right)\right) \quad \text { for } \quad 0 \leq t \leq 1 .
$$

Using Lemma 4.4 with $\beta=1$ and $B=|D|$, we get

$$
\widetilde{\varphi}(t)=u_{|D|}^{+}(t)+i I u_{|D|}^{-}(t) \quad \text { for } \quad t \in \mathbb{R} .
$$

(a) We define $f_{1}: \mathbb{R}_{\tau} \rightarrow B\left(V_{\mathbb{C}}\right)$ by $f_{1}\left(t, \tau^{\varepsilon}\right):=u_{|D|}^{+}(t)$ for $t \in \mathbb{R}, \varepsilon \in\{0,1\}$. To see that $f_{1}$ is positive definite, it suffices to verify this for its restriction to $\mathbb{R}$ (Lemma 3.6), which follows from the positivity of the Fourier coefficients in the expansion

$$
u_{|D|}^{+}(t)=\sum_{n \in \mathbb{Z}} c_{2 n}^{|D|} e^{2 n \pi i t} \quad \text { with } \quad c_{2 n}^{|D|}=\frac{\mathbf{1}-e^{-|D|}}{\mathbf{1}+e^{-|D|}} \frac{2|D|}{|D|^{2}+(2 n \pi)^{2} \mathbf{1}} \geq 0
$$

(Lemma 4.4). Note that $f_{1}$ is 1 -periodic.

(b) Likewise, the function $f_{2}: \mathbb{R}_{\tau} \rightarrow B\left(V_{\mathbb{C}}\right)$ defined by $f_{2}\left(t, \tau^{\varepsilon}\right):=u_{|D|}^{-}(t)$ for $t \in \mathbb{R}, \varepsilon \in\{0,1\}$ is positive definite because the Fourier coefficients

$$
c_{2 n+1}^{|D|}=\frac{2|D|}{|D|^{2}+((2 n+1) \pi)^{2} \mathbf{1}} \geq 0 \quad \text { for } \quad n \in \mathbb{Z}
$$

are positive. Note that $f_{2}\left(t+1, \tau^{\varepsilon}\right)=-f_{2}\left(t, \tau^{\varepsilon}\right)$ for $t \in \mathbb{R}, \varepsilon \in\{0,1\}$.

(c) We now consider the function

$$
\widetilde{f}_{2}(g):=h(g) f_{2}(g) \quad \text { with } \quad h\left(t, \tau^{\varepsilon}\right)=(i I)^{\varepsilon} \quad \text { for } \quad t \in \mathbb{R}, \varepsilon \in\{0,1\} .
$$

Since $h(g)$ commutes with $f_{2}\left(g^{\prime}\right)$ for $g, g^{\prime} \in \mathbb{R}_{\tau}$, the function $\widetilde{f}_{2}$ is positive definite if $h$ is positive definite (Lemma A.6). As $h$ is constant on the two $\mathbb{R}$-cosets and its restriction to the 2-element subgroup $\{\mathbf{1}, \tau\}$ is a unitary representation, $h$ is positive definite. We conclude that the $B\left(V_{\mathbb{C}}\right)$-valued function $f:=f_{1}+\widetilde{f}_{2}$ on $\mathbb{R}_{\tau}$ is positive definite. 
Corollary 4.6. Let $V$ be a real vector space and let $\psi: \mathbb{R} \rightarrow \operatorname{Bil}(V)$ be a continuous positive definite function satisfying the $\beta$-KMS condition. Then there exists a pointwise continuous function $f: \mathbb{R}_{\tau} \rightarrow$ $\operatorname{Bil}(V)$ which is reflection positive with respect to the subset $[0, \beta / 2] \subseteq \mathbb{R}$ and which satisfies

$$
f(t, \tau)=\psi(i t) \quad \text { for } \quad 0 \leq t \leq \beta \quad \text { and } \quad f(t+\beta, \tau)=\overline{f(t, \tau)} \quad \text { for } \quad t \in \mathbb{R} .
$$

Remark 4.7. The function $\widetilde{f}_{2}$ in the proof of Theorem 4.5 is not reflection positive because $\widetilde{f}_{2}(\beta, \tau)$ is a negative operator. This also shows that the natural decomposition $f=f_{1}+\widetilde{f}_{2}$ into even and odd part is not compatible with reflection positivity.

\subsection{Integral representation of reflection positive functions}

We now describe an integral representation of the reflection positive function $f: \mathbb{R}_{\tau} \rightarrow \operatorname{Bil}(V)$ which corresponds to the decomposition of the corresponding unitary representation of $\mathbb{R}_{\tau}$. With

$$
\widetilde{\varphi}(t)=(\mathbf{1}+i C)^{1-t / \beta}(\mathbf{1}-i C)^{t / \beta} \quad \text { for } \quad 0 \leq t \leq \beta,
$$

where $C \in B(V)$ is a skew-symmetric strict contraction, we first decompose $V$ into $V_{0}:=\operatorname{ker} C$ and $V_{1}:=V_{0}^{\perp}=\overline{C V}$. Then the polar decomposition $C=I|C|$ yields a complex structure $I$ on $V_{1}$. Accordingly, we write $\widetilde{\varphi}=\widetilde{\varphi}_{0}+\widetilde{\varphi}_{1}$, where $\widetilde{\varphi}_{0}=\mathbf{1}$ is constant. This component leads to the constant function $f_{0}(t, \tau)=\mathbf{1}$. We now assume that $V=V_{1}$, i.e., that $C$ is injective. Then $I$ is a complex structure on $V$.

Proposition 4.8. If $\operatorname{ker} C=\{0\}$ and $P$ denotes the spectral measure of the symmetric operator $|D|=$ $\frac{1}{\beta} \log \frac{1+|C|}{1-|C|}$ on $V$, then we have the integral representation

$$
f\left(t, \tau^{\varepsilon}\right)=\int_{(0, \infty)} u_{\lambda}^{+}(t)+u_{\lambda}^{-}(t)(i I)^{\varepsilon} d P(\lambda)
$$

where $u_{\lambda}^{ \pm}: \mathbb{R} \rightarrow \mathbb{R}$ are defined by $u_{\lambda}^{ \pm}(t+\beta)= \pm u_{\lambda}^{ \pm}(t)$ and

$$
u_{\lambda}^{ \pm}(t):=\frac{e^{-t \lambda} \pm e^{-(\beta-t) \lambda}}{1+e^{-\beta \lambda}} \quad \text { for } \quad 0 \leq t \leq \beta .
$$

Proof. First we observe that $|D|$ is a positive symmetric operator with trivial kernel which commutes with $I$. We therefore have $|D|=\int_{(0, \infty)} \lambda d P(\lambda)$. With the notation from Lemma 4.4, we then have

$$
f\left(t, \tau^{\varepsilon}\right)=u_{|D|}^{+}(t)+u_{|D|}^{-}(t)(i I)^{\varepsilon} \quad \text { for } \quad t \in \mathbb{R}, \varepsilon \in\{0,1\} .
$$

¿From the integral representations $u_{|D|}^{ \pm}(t)=\int_{(0, \infty)} u_{\lambda}^{ \pm}(t) d P(\lambda)$, we now obtain (19)).

Remark 4.9. (a) For $0 \leq t \leq \beta$, we have in particular

$$
f\left(t, \tau^{\varepsilon}\right)=\int_{(0, \infty)} \frac{e^{-t \lambda}+e^{-(\beta-t) \lambda}}{1+e^{-\beta \lambda}} \mathbf{1}+\frac{e^{-t \lambda}-e^{-(\beta-t) \lambda}}{1+e^{-\beta \lambda}}(i I)^{\varepsilon} d P(\lambda)
$$

(b) The most basic type is obtained for $D=\lambda \mathbf{1}, \lambda>0$, which leads to

$$
f\left(t, \tau^{\varepsilon}\right)=\frac{\left(e^{-t \lambda}+e^{-(\beta-t) \lambda}\right) \mathbf{1}+\left(e^{-t \lambda}-e^{-(\beta-t) \lambda}\right)(i I)^{\varepsilon}}{1+e^{-\beta \lambda}}=u_{\lambda}^{+}(t) \mathbf{1}+u_{\lambda}^{-}(t)(i I)^{\varepsilon} \quad \text { for } \quad 0 \leq t \leq \beta .
$$


The simplest non-trivial example arises for $V=\mathbb{R}^{2}$ with $I=\left(\begin{array}{cc}0 & -1 \\ 1 & 0\end{array}\right)$.

(c) Every Borel spectral measure $P$ on $(0, \infty)$ which commutes with $I$ defines a positive operator $|D|=\int_{0}^{\infty} \lambda d P(\lambda)$ and we may put $D:=-I|D|$. Then $\operatorname{ker}|D|=0$, so that

$$
|C|:=\frac{e^{\beta|D|}-\mathbf{1}}{e^{\beta|D|}+\mathbf{1}}=\tanh \left(\frac{\beta|D|}{2}\right)
$$

is a symmetric contraction with trivial kernel commuting with $I$, and therefore $C:=I|C|$ is a skewsymmetric contraction with polar decomposition $I|C|$ and $|D|=\frac{1}{\beta} \log \left(\frac{\mathbf{1}+|C|}{\mathbf{1}-|C|}\right)$.

\subsection{Characterizing reflection positive extensions}

In Theorem 4.5 we obtained positive definite extensions to all or $\mathbb{R}_{\tau}$ for certain functions on the coset $\mathbb{R} \rtimes\{\tau\}$. In this section we describe an intrinsic characterization of those weakly continuous reflection positive functions $f: \mathbb{R}_{\tau} \rightarrow B\left(V_{\mathbb{C}}\right)$ arising from this construction. First we observe that we can recover $\psi$ from $f$ :

Lemma 4.10. If $f: \mathbb{R}_{\tau} \rightarrow \operatorname{Bil}(V)$ is reflection positive and pointwise continuous, then there exists a unique $\beta$-KMS positive definite function $\psi: \mathbb{R} \rightarrow \operatorname{Bil}(V)$ with

$$
f(t, \tau)=\psi(i t) \quad \text { for } \quad 0 \leq t \leq \beta .
$$

Proof. First we observe that the function $\varphi(t):=f(t, \tau)$ has values in $\operatorname{Herm}\left(V_{\mathbb{C}}\right)$ and satisfies

$$
\varphi(t+\beta)=\overline{\varphi(t)} \quad \text { for } \quad t \in \mathbb{R} .
$$

Reflection positivity implies that the kernel $\varphi\left(\frac{t+s}{2}\right)$ for $0 \leq t, s \leq \beta$ is positive definite. By NÓ15, Thm. B.3], there exists a $\operatorname{Bil}^{+}(V)$-valued measure $\mu$ such that

$$
\varphi(t)=\int_{\mathbb{R}} e^{-\lambda t} d \mu(\lambda) \quad \text { for } \quad 0<t<\beta .
$$

The continuity of $\varphi$ on $[0, \beta]$ actually implies that the integral representation also holds on the closed interval $[0, \beta]$ by the Monotone Convergence Theorem. In particular, the measure $\mu$ is finite. Therefore its Fourier transform $\psi(t):=\int_{\mathbb{R}} e^{i t \lambda} d \mu(\lambda)$ is a pointwise continuous $\operatorname{Bil}(V)$-valued positive definite function on $\mathbb{R}$. Further, (20) implies

$$
e^{\beta \lambda} d \mu(-\lambda)=d \bar{\mu}(\lambda)
$$

and Theorem 4.5 shows that $\varphi(t)=\psi(i t)$ holds for the $\beta$-KMS function $\psi: \mathbb{R} \rightarrow \operatorname{Bil}(V)$.

Before we describe a realization of the GNS representation $\left(U^{f}, \mathcal{H}_{f}\right)$ in spaces of sections of a vector bundle, let us recall the general background for this.

Remark 4.11. For a $B(V)$-valued positive definite function $f: G \rightarrow B(V)$, the reproducing kernel Hilbert space with kernel $K(g, h)=\varphi\left(g h^{-1}\right)=K_{g} K_{h}^{*}$ is generated by the functions

$$
K_{h, w}:=K_{h}^{*} w \quad \text { with } \quad K_{h, w}(g)=K_{g} K_{h}^{*} w=K(g, h) w=\varphi\left(g h^{-1}\right) w .
$$


The group $G$ acts on this space by right translations

$$
\left(U_{g} s\right)(h):=s(h g) .
$$

If $P \subseteq G$ is a subgroup and $(\rho, V)$ is a unitary representation for which

$$
f(h g)=\rho(h) f(g) \quad \text { for all } \quad g \in G, h \in P,
$$

then

$$
\mathcal{H}_{f} \subseteq \mathcal{F}(G, V)_{\rho}:=\{s: G \rightarrow V:(\forall g \in G)(\forall h \in P) s(h g)=\rho(h) s(g)\} .
$$

Therefore $\mathcal{H}_{f}$ can be identified with a space of sections of the associated bundle

$$
\mathbb{V}:=\left(V \times{ }_{P} G\right)=(V \times G) / P,
$$

where $P$ acts on the trivial vector bundle $V \times G$ over $G$ by $h .(v, g)=(\rho(h) v, h g)$.

To derive a suitable characterization of the functions $f$ arising in Theorem 4.5, we identify $2 \beta$-periodic function $s$ on $\mathbb{R}$ with pairs of function $\left(s_{0}, s_{1}\right)$ via $s=s_{0}+s_{1}$, where $s_{0}$ is $\beta$-periodic and $s_{1}(\beta+t)=-s_{1}(t)$. Accordingly, any $2 \beta$-periodic function $s: \mathbb{R} \rightarrow V_{\mathbb{C}}$ defines a function

$$
\widetilde{s}: \mathbb{R} \rightarrow V_{\mathbb{C}}^{2}, \quad \widetilde{s}=\left(s_{1}, s_{2}\right) \quad \text { with } \quad \widetilde{s}(\beta+t)=\left(\begin{array}{cc}
\mathbf{1} & 0 \\
0 & -\mathbf{1}
\end{array}\right) \widetilde{s}(t) .
$$

In this sense $\widetilde{s}$ is a section of the vector bundle over $\mathbb{T}_{\beta}$ with fiber $V_{\mathbb{C}}^{2}$ defined by the representation of $\beta \mathbb{Z}$, specified by

$$
\rho(\beta)=\left(\begin{array}{cc}
\mathbf{1} & 0 \\
0 & -\mathbf{1}
\end{array}\right) .
$$

Splitting the $B(V)$-valued positive definite function

$$
f: \mathbb{R}_{\tau} \rightarrow B(V), \quad f\left(t, \tau^{\varepsilon}\right)=u_{|D|}^{+}(t)+u_{|D|}^{-}(t)(i I)^{\varepsilon} \quad \text { for } \quad t \in \mathbb{R}, \varepsilon \in\{0,1\}
$$

into even and odd part with respect to the $\beta$-translation, we obtain:

Lemma 4.12. For the subgroup $P:=(\mathbb{Z} \beta)_{\tau} \cong \mathbb{Z} \beta \rtimes\{\mathbf{1}, \tau\}$ of $G:=\mathbb{R}_{\tau}$, we consider the unitary representation $\rho: P \rightarrow \mathrm{U}\left(V_{\mathbb{C}}^{2}\right)$ defined by

$$
\rho(\beta, \mathbf{1}):=\left(\begin{array}{cc}
\mathbf{1} & 0 \\
0 & -\mathbf{1}
\end{array}\right) \quad \text { and } \quad \rho(0, \tau):=\left(\begin{array}{cc}
\mathbf{1} & 0 \\
0 & i I
\end{array}\right),
$$

where $I$ is a complex structure on the real Hilbert space $V$ commuting with the positive operator $|D|$. Then

$$
f^{\sharp}: \mathbb{R}_{\tau} \rightarrow B\left(V^{2}\right) \cong M_{2}(B(V)), \quad f^{\sharp}\left(t, \tau^{\varepsilon}\right):=\left(\begin{array}{cc}
u_{|D|}^{+}(t) & 0 \\
0 & u_{|D|}^{-}(t)(i I)^{\varepsilon}
\end{array}\right)
$$

is a positive definite function satisfying

$$
f^{\sharp}(h g)=\rho(h) f^{\sharp}(g) \quad \text { for } \quad h \in P, g \in G .
$$

The corresponding GNS representation $\left(U^{f^{\sharp}}, \mathcal{H}_{f^{\sharp}}\right)$ is equivalent to the GNS representation $\left(U^{f}, \mathcal{H}_{f}\right)$. 
Proof. The first assertion follows from

$$
f^{\sharp}\left((0, \tau)\left(t, \tau^{\varepsilon}\right)\right)=f^{\sharp}\left(-t, \tau^{\varepsilon+1}\right)=\left(\begin{array}{cc}
u_{|D|}^{+}(-t) & 0 \\
0 & u_{|D|}^{-}(-t)(i I)^{\varepsilon+1}
\end{array}\right)=\left(\begin{array}{cc}
u_{|D|}^{+}(t) & 0 \\
0 & u_{|D|}^{-}(t)(i I)^{\varepsilon+1}
\end{array}\right)
$$

and

$$
f^{\sharp}\left(\beta+t, \tau^{\varepsilon}\right)=\left(\begin{array}{cc}
u_{|D|}^{+}(t) & 0 \\
0 & -u_{|D|}^{-}(t)(i I)^{\varepsilon}
\end{array}\right) .
$$

As the GNS representation $\left(U^{f}, \mathcal{H}_{f}\right)$ decomposes under the involution $U_{\beta}^{f}$ into \pm 1 -eigenspaces, this representation is equivalent to the GNS representation $\left(U^{f^{\sharp}}, \mathcal{H}_{f^{\sharp}}\right)$ corresponding to $f^{\sharp}$.

Remark 4.13. (a) The preceding lemma implies that, if the complex structure $I$ on $V$ is fixed, then the relation (23) determines $f^{\sharp}$ completely in terms of the function

$$
[0, \beta] \rightarrow M_{2}(B(V)), \quad t \mapsto f^{\sharp}(t, \tau)=\left(\begin{array}{cc}
\operatorname{Re} \varphi(t) & 0 \\
0 & i \operatorname{Im} \varphi(t)
\end{array}\right),
$$

so that $\varphi$ determines $f$ in a natural way.

(b) This lemma also shows that we may identify the Hilbert space $\mathcal{H}_{f} \cong \mathcal{H}_{f \sharp}$ as a space of section of a Hilbert bundle $V^{2} \times{ }_{\rho} G$ over the circle $\mathbb{T}_{\beta} \cong \mathbb{R}_{\tau} / H$ with fiber $V^{2}$.

(c) Every function $s: \mathbb{R}_{\tau} \rightarrow V^{2}$ satisfying $s(h g)=\rho(h) s(g)$ for $h \in(\beta \mathbb{Z})_{\tau}$ is determined by its restriction $\widetilde{s}$ to the subgroup $\mathbb{R}$, which satisfies

$$
\widetilde{s}(\beta+t)=\rho(\beta, \mathbf{1}) \widetilde{s}(t) \quad \text { for } \quad t \in \mathbb{R} .
$$

The action of $\tau$ is in this picture given by

$$
(\tau . \widetilde{s})(t):=s(t, \tau)=s((0, \tau)(-t, \mathbf{1}))=\rho(\tau) \widetilde{s}(-t) .
$$

Remark 4.14. (a) In view of (22), there exists a $\operatorname{Bil}^{+}(V)$-valued measure $\nu$ on $[0, \infty)$ for which we can write

$$
d \mu(\lambda)=d \nu(\lambda)+e^{\beta \lambda} d \bar{\nu}(-\lambda) .
$$

This leads for $0 \leq t \leq \beta$ and $\nu=\nu_{1}+i \nu_{2}$ to

$$
\varphi(t)=\int_{0}^{\infty} e^{-t \lambda}+e^{-(\beta-t) \lambda} d \nu_{1}(\lambda)+i \int_{0}^{\infty} e^{-t \lambda}-e^{-(\beta-t) \lambda} d \nu_{2}(\lambda) .
$$

In particular, the most elementary non-trivial examples correspond to the Dirac measures of the form $\nu=\delta_{\lambda} \cdot(\gamma+i \omega)$, where $\delta_{\lambda}$ is the Dirac measure in $\lambda>0$ :

$\varphi(t)=\left(e^{-t \lambda}+e^{-(\beta-t) \lambda}\right) \gamma+i\left(e^{-t \lambda}-e^{-(\beta-t) \lambda}\right) \omega=e^{-t \lambda} h+e^{-(\beta-t) \lambda} \bar{h}, \quad$ where $\quad h:=\gamma+i \omega \in \operatorname{Bil}^{+}(V)$.

Writing $\omega(v, w)=\gamma(v, C w)$ (Corollary A.9) and replacing $V$ by the real Hilbert space defined by the positive semidefinite form $\gamma$ on $V$, we obtain the $B\left(V_{\mathbb{C}}\right)$-valued function

$$
\widetilde{\varphi}(t)=\left(e^{-t \lambda}+e^{-(\beta-t) \lambda}\right)+i C\left(e^{-t \lambda}-e^{-(\beta-t) \lambda}\right)=e^{-t \lambda}(\mathbf{1}+i C)+e^{-(\beta-t) \lambda}(\mathbf{1}-i C) \quad \text { for } \quad 0 \leq t \leq \beta,
$$


which leads to

$$
f\left(t, \tau^{\varepsilon}\right)=\left(1+e^{-\beta \lambda}\right)\left(u_{\lambda}^{+}(t) \mathbf{1}+u_{\lambda}^{-}(t)|C|(i I)^{\varepsilon}\right) \quad \text { for } \quad t \in \mathbb{R}, \varepsilon \in\{0,1\} .
$$

(b) This can also be formulated in terms of forms. With $\gamma(v, w)=\langle v, w\rangle_{V}$ and

$$
h(v, w)=\gamma(v, w)+i \omega(v, w)=\langle v,(\mathbf{1}+i C) w\rangle_{V_{\mathbb{C}}}=\langle v,(\mathbf{1}+i I|C|) w\rangle_{V_{\mathbb{C}}},
$$

we get

$$
f\left(t, \tau^{\varepsilon}\right)(v, w)=\left\langle v,\left(u_{\lambda}^{+}(t) \mathbf{1}+u_{\lambda}^{-}(t)|C|(i I)^{\varepsilon}\right) w\right\rangle .
$$

\subsection{Realization by resolvents of the Laplacian}

We have seen in the preceding subsection how to obtain a realization of the Hilbert space $\mathcal{H}_{f}$ as a space $\mathcal{H}_{f^{\sharp}}$ of sections of a Hilbert bundle $\mathbb{V}$ with fiber $V_{\mathbb{C}}^{2}$ over the circle $\mathbb{T}_{\beta}=\mathbb{R} / \beta \mathbb{Z}$. In this section we provide an analytic description of the scalar product on this space if $|D|=\lambda \mathbf{1}$ for some $\lambda>0$. We shall see that it has a natural description in terms of the resolvent $\left(\lambda^{2}-\Delta\right)^{-1}$ of the Laplacian of $\mathbb{T}_{\beta}$ acting on section of the bundle $\mathbb{V}$.

On the circle group $\mathbb{T}_{2 \beta}$, we consider the normalized Haar measure given by

$$
\int_{\mathbb{T}_{2 \beta}} h(t) d \mu_{\mathbb{T}_{2 \beta}}=\frac{1}{2 \beta} \int_{0}^{2 \beta} h(t) d t
$$

where we identify functions $h$ on $\mathbb{T}_{2 \beta}$ with $2 \beta$-periodic functions on $\mathbb{R}$.

As in Lemma 4.12, we write

$$
f^{\sharp}\left(t, \tau^{\varepsilon}\right)=\left(\begin{array}{cc}
u_{\lambda}^{+}(t) \mathbf{1} & 0 \\
0 & u_{\lambda}^{-}(t)(i I)^{\varepsilon}
\end{array}\right) \in B\left(V_{\mathbb{C}}^{2}\right) \cong M_{2}\left(B\left(V_{\mathbb{C}}\right)\right),
$$

For $\chi_{n}(t)=e^{\pi i n t / \beta}$ we then have

$$
u_{\lambda}^{+}=\sum_{n \in \mathbb{Z}} c_{2 n}^{\lambda} \chi_{2 n} \quad \text { and } \quad u_{\lambda}^{-}=\sum_{n \in \mathbb{Z}} c_{2 n+1}^{\lambda} \chi_{2 n+1},
$$

where

$$
c_{n}^{\lambda}=c_{-n}^{\lambda}=\frac{1-(-1)^{n} e^{-\beta \lambda}}{1+e^{-\beta \lambda}} \cdot \frac{2 \beta \lambda}{(\beta \lambda)^{2}+(n \pi)^{2}}=\frac{1-(-1)^{n} e^{-\beta \lambda}}{1+e^{-\beta \lambda}} \cdot \frac{2 \lambda}{\beta} \cdot \frac{1}{\lambda^{2}+(n \pi / \beta)^{2}} \quad \text { for } \quad n \in \mathbb{Z}
$$

(the rightmost factors are called bosonic Matsubara coefficients if $n$ is even and fermionic if $n$ is odd [DG13, §18]). With

$$
c_{+}^{\lambda}:=\frac{1-e^{-\beta \lambda}}{1+e^{-\beta \lambda}} \frac{2 \lambda}{\beta}=\tanh \left(\frac{\beta \lambda}{2}\right) \frac{2 \lambda}{\beta} \quad \text { and } \quad c_{-}^{\lambda}:=\frac{2 \lambda}{\beta},
$$

we thus obtain

$$
c_{2 n}^{\lambda}=\frac{c_{+}^{\lambda}}{\lambda^{2}+(2 n \pi / \beta)^{2}}, \quad c_{2 n+1}^{\lambda}=\frac{c_{-}^{\lambda}}{\lambda^{2}+((2 n+1) \pi / \beta)^{2}} .
$$

The following proposition shows that the positive operator $\left(\lambda^{2}-\Delta\right)^{-1}$ on the Hilbert space of $L^{2}$ section of $\mathbb{V}$ defines a unitary representation of $\mathbb{R}_{\tau}$ which is unitarily equivalent to the representation on $\mathcal{H}_{f}$ (cf. Lemma 4.12). 
Proposition 4.15. For $\lambda>0$, let $\mathcal{H}_{\lambda}$ be the Hilbert space obtained by completing the space

$$
\Gamma_{\rho}:=\left\{s \in C^{\infty}\left(\mathbb{R}_{\tau}, V_{\mathbb{C}}^{2}\right):\left(\forall g \in \mathbb{R}_{\tau}, h \in(\mathbb{Z} \beta)_{\tau}\right) s(h g)=\rho(h) s(g)\right\}
$$

with respect to

$$
\left\langle s_{1}, s_{2}\right\rangle:=\frac{1}{2 \beta} \int_{0}^{2 \beta}\left\langle s_{1}(t, \mathbf{1}),\left(\left(\lambda^{2}-\Delta\right)^{-1} s_{2}\right)(t, \mathbf{1})\right\rangle d t .
$$

On $\mathcal{H}_{\lambda}$ we have a natural unitary representation $U^{\lambda}$ of $\mathbb{R}_{\tau}$ by right translation which is unitarily equivalent to the GNS representation $\left(U^{f^{\sharp}}, \mathcal{H}_{f^{\sharp}}\right)$. Here the corresponding $j$-map is given by

$$
j: V \rightarrow \mathcal{H}_{\lambda}, \quad j\left(\begin{array}{l}
v_{1} \\
v_{2}
\end{array}\right)=\sqrt{c_{+}^{\lambda}} \sum_{n \in \mathbb{Z}} \chi_{2 n}\left(\begin{array}{c}
v_{1} \\
0
\end{array}\right)+\sqrt{c_{-}^{\lambda}} \sum_{n \in \mathbb{Z}} \chi_{2 n+1}\left(\begin{array}{c}
0 \\
v_{2}
\end{array}\right) .
$$

Proof. We identify $\Gamma_{\rho}$ with the space

$$
\left\{s \in C^{\infty}\left(\mathbb{R}, V_{\mathbb{C}}^{2}\right):(\forall t \in \mathbb{R}) s(\beta+t)=\rho(\beta) s(t)\right\}
$$

(Remark 4.13). Then $s=\left(\begin{array}{l}s_{+} \\ s_{-}\end{array}\right)$, where $s_{+}$is $\beta$-periodic and $s_{-}$is $\beta$-antiperiodic. Accordingly, we have an orthogonal decomposition $\mathcal{H}_{\lambda}=\mathcal{H}_{\lambda}^{+} \oplus \mathcal{H}_{\lambda}^{-}$, where $\mathcal{H}_{\lambda}^{ \pm}=\left\{s \in \mathcal{H}_{\lambda}:(\forall t \in \mathbb{R}) s(\beta+t)= \pm s(t)\right\}$. Then $U^{\lambda}$ is given by

$$
\left(U_{t}^{\lambda} s\right)(x)=s(t+x) \quad \text { for } \quad t, x \in \mathbb{R} \quad \text { and } \quad\left(U_{\tau}^{\lambda} s\right)(x)=\left(\begin{array}{c}
s_{+}(-x) \\
(i I) s_{-}(-x)
\end{array}\right) .
$$

¿From the Fourier expansion $s=\sum_{n \in \mathbb{Z}} \chi_{n} s_{n}$ and the orthonormality of the $\chi_{n}$, we then derive

$$
\left\langle s_{1}, s_{2}\right\rangle_{\mathcal{H}}=\sum_{n \in \mathbb{Z}} \frac{\left\langle s_{1, n}, s_{2, n}\right\rangle}{\lambda^{2}+(n \pi / \beta)^{2}} .
$$

For the map $j: V \rightarrow \mathcal{H}_{\lambda}$ in (29), the image is $U_{\mathbb{R}}^{\lambda}$-generating for $\mathcal{H}_{\lambda}$ because the projection onto each Fourier component generates the first, resp., the second component of $V_{\mathbb{C}}^{2}$, according to parity. Therefore the unitary representation $\left(U^{\lambda}, \mathcal{H}_{\lambda}\right)$ is equivalent to the GNS representation of the positive definite function $\widetilde{f}: \mathbb{R}_{\tau} \rightarrow B\left(V_{\mathbb{C}}^{2}\right)$, given by

¿From

$$
\langle\mathbf{v}, \widetilde{f}(g) \mathbf{w}\rangle=\left\langle j(\mathbf{v}), U_{g}^{\tau} j(\mathbf{w})\right\rangle_{\mathcal{H}_{\lambda}} .
$$

$$
U_{\left(t, \tau^{\varepsilon}\right)}^{\lambda} j(\mathbf{v})=\sqrt{c_{+}^{\lambda}} \sum_{n \in \mathbb{Z}} \chi_{2 n} \chi_{2 n}(t)\left(\begin{array}{c}
v_{1} \\
0
\end{array}\right)+\sqrt{c_{-}^{\lambda}} \sum_{n \in \mathbb{Z}} \chi_{2 n+1} \chi_{2 n+1}(t)\left(\begin{array}{c}
0 \\
(i I)^{\varepsilon} v_{2}
\end{array}\right),
$$

we derive with (28)

$$
\begin{aligned}
\left\langle\mathbf{v}, \widetilde{f}\left(t, \tau^{\varepsilon}\right) \mathbf{w}\right\rangle & =c_{+}^{\lambda} \sum_{n \in \mathbb{Z}} \frac{\chi_{2 n}(t)}{\lambda^{2}+(2 n \pi / \beta)^{2}}\left\langle v_{1}, w_{1}\right\rangle+c_{-}^{\lambda} \sum_{n \in \mathbb{Z}} \frac{\chi_{2 n+1}(t)}{\lambda^{2}+((2 n+1) \pi / \beta)^{2}}\left\langle v_{1},(i I)^{\varepsilon} w_{2}\right\rangle \\
& =\sum_{n \in \mathbb{Z}} \chi_{2 n}(t) c_{2 n}^{\lambda}\left\langle v_{1}, w_{1}\right\rangle+\sum_{n \in \mathbb{Z}} \chi_{2 n+1}(t) c_{2 n+1}^{\lambda}\left\langle v_{2},(i I)^{\varepsilon} w_{2}\right\rangle \\
& =\left\langle v_{1}, u_{\lambda}^{+}(t) w_{1}\right\rangle+\left\langle v_{2}, u_{\lambda}^{-}(t)(i I)^{\varepsilon} w_{2}\right\rangle=\left\langle\mathbf{v}, f^{\sharp}\left(t, \tau^{\varepsilon}\right) \mathbf{w}\right\rangle .
\end{aligned}
$$

This shows that $\tilde{f}=f^{\sharp}$, which completes the proof. 
Remark 4.16. From $u_{\lambda}^{+}=\sum_{n \in \mathbb{Z}} c_{2 n}^{\lambda} \chi_{2 n}$ it follows that

$$
\left(\lambda^{2}-\Delta\right) u_{\lambda}^{+}=\sum_{n \in \mathbb{Z}} c_{2 n}^{\lambda}\left(\lambda^{2}+\frac{(2 \pi n)^{2}}{\beta^{2}}\right) \chi_{2 n}=c_{+}^{\lambda} \sum_{n \in \mathbb{Z}} \chi_{2 n}=c_{+}^{\lambda} \delta_{0},
$$

where the latter relation means that

$$
s_{+}(0)=\frac{1}{2 \beta} \sum_{n \in \mathbb{Z}} \int_{0}^{2 \beta} s_{+}(t) \chi_{2 n}(t) d t
$$

for every smooth $\beta$-periodic functions $s_{+}$on $\mathbb{R}$. This relation can also be written as

$$
\left(\lambda^{2}-\Delta\right)^{-1} \delta_{0}=\frac{1}{c_{+}^{\lambda}} u_{\lambda}^{+} .
$$

¿From $u_{\lambda}^{-}=\sum_{n \in \mathbb{Z}} c_{2 n+1}^{\lambda} \chi_{2 n+1}$ it follows that

$$
\left(\lambda^{2}-\Delta\right) u_{\lambda}^{-}=\sum_{n \in \mathbb{Z}} c_{2 n+1}^{\lambda}\left(\lambda^{2}+\frac{(2 n+1)^{2} \pi^{2}}{\beta^{2}}\right) \chi_{2 n+1}=c_{-}^{\lambda} \chi_{1} \sum_{n \in \mathbb{Z}} \chi_{2 n}
$$

As every smooth $\beta$-antiperiodic function $s_{-}$is of the form $s_{-}=\chi_{-1} s_{+}$, where $s_{+}$is $\beta$-periodic, we obtain, in the sense of distributions,

$$
\left\langle\left(\lambda^{2}-\Delta\right) u_{\lambda}^{-}, s_{-}\right\rangle=c_{-}^{\lambda} s_{+}(0)=c_{-}^{\lambda} s_{-}(0)=\left\langle c_{-}^{\lambda} \delta_{0}, s_{-}\right\rangle,
$$

and therefore

$$
\left(\lambda^{2}-\Delta\right)^{-1} \delta_{0}=\frac{1}{c_{-}^{\lambda}} u_{\lambda}^{-}
$$

on $\beta$-antiperiodic functions. Combining all this, we get

$$
\left(\left(\lambda^{2}-\Delta\right) f^{\sharp}\right)\left(t, \tau^{\varepsilon}\right)=\left(\begin{array}{cc}
\left(\lambda^{2}-\Delta\right) u_{\lambda}^{+} \mathbf{1} & 0 \\
0 & \left(\lambda^{2}-\Delta\right) u_{\lambda}^{-}(i I)^{\varepsilon}
\end{array}\right)=\delta_{0}\left(\begin{array}{cc}
c_{+}^{\lambda} \mathbf{1} & 0 \\
0 & c_{-}^{\lambda}(i I)^{\varepsilon}
\end{array}\right)
$$

as an operator-valued distribution on the space of smooth sections of $\mathbb{V}$ (cf. also the discussion of thermal euclidean Green's functions in [DG13, Def. 18.49]).

\section{The case $\beta=\infty$}

In the context of $C^{*}$-dynamical systems, it is well known that that the positive energy condition for the unitary one-parameter group implementing the automorphisms of a $C^{*}$-algebra $\mathcal{A}$ in a representation can be viewed as a KMS condition for $\beta=\infty$ (cf. BR96]). For reflection positive representations of $G=\mathbb{R}$, this case corresponds to $G_{+}=\mathbb{R}_{+}$, which has been treated in [NÓ14a, NÓ14b] (cf. also the discussion of euclidean Green's functions in [DG13, Def. 18.48]). The following theorem makes this analogy also transparent in the context of our Theorem 2.6.

If $\psi: \mathbb{R} \rightarrow \operatorname{Bil}(V)$ is a positive definite function satisfying the KMS condition for $\beta>0$, then its extension to $\overline{\mathcal{S}_{\beta}}$ is pointwise bounded (Theorem 2.6). This observation explains the assumptions in the following theorem. 
Theorem 5.1. (KMS condition for $\beta=\infty$ ) Let $V$ be a real vector space and let $\psi: \mathbb{R} \rightarrow \operatorname{Bil}(V)$ be a pointwise continuous positive definite function. Then the following are equivalent:

(i) $\psi$ extends to a pointwise bounded function on the closed upper half plane which is pointwise holomorphic on $\mathbb{C}_{+}$.

(ii) There exists a $\operatorname{Bil}^{+}(V)$-valued regular Borel measure $\mu$ on $[0, \infty)$ satisfying

$$
\psi(t)=\int_{0}^{\infty} e^{i t \lambda} d \mu(\lambda)
$$

(iii) The GNS representation $\left(U^{\psi}, \mathcal{H}_{\psi}\right)$ has spectrum contained in $[0, \infty)$.

If this is the case, then the function

$$
f\left(t, \tau^{\varepsilon}\right):=\psi(i|t|) \quad \text { for } \quad t \in \mathbb{R}, \varepsilon \in\{0,1\},
$$

on $\mathbb{R}_{\tau}$ is reflection positive with respect to $\mathbb{R}_{+}=[0, \infty)$.

Proof. (i) $\Rightarrow$ (ii): First we use NÓ15, Prop. B.1] to write $\varphi$ as the Fourier transform of a $\mathrm{Bil}^{+}(V)$-valued regular Borel measure $\mu$ on $\mathbb{R}: \psi(t)=\int_{\mathbb{R}} e^{i t \lambda} d \mu(\lambda)$. Evaluating in $v \in V_{\mathbb{C}}$, we obtain for the positive measure $\mu^{v, v}:=\mu(\cdot)(v, v)$ the relation

$$
\psi(t)(v, v)=\int_{\mathbb{R}} e^{i t \lambda} d \mu^{v, v}(\lambda) .
$$

This function extends to a bounded holomorphic function $\psi$ on $\mathbb{C}_{+}$. In particular, the Laplace transform $\mathcal{L}\left(\mu^{v, v}\right)(t)=\psi(i t)(v, v)$ is bounded, which implies that $\operatorname{supp}\left(\mu^{v, v}\right) \subseteq[0, \infty)$ (cf. [Ne00, Rem. V.4.12]). This implies that $\mu$ is supported on $[0, \infty)$.

(ii) $\Rightarrow$ (iii): Write $U_{t}:=U_{t}^{\psi}=e^{i t H}$ with the selfadjoint generator $H$. We show that $H \geq 0$. Let $E$ be the spectral measure of $H$, so that $H=\int_{\mathbb{R}} \lambda d E(\lambda)$ and $U_{t}=\int_{\mathbb{R}} e^{i t \lambda} d E(\lambda)$. It suffices to show that, for every $f \in L^{1}(\mathbb{R})$ for which the Fourier transform $\widehat{f}(\lambda)=\int_{\mathbb{R}} e^{i \lambda t} f(t) d t$ vanishes on $\mathbb{R}_{+}$, the operator

$$
U_{f}=\int_{\mathbb{R}} f(t) e^{i t H} d t=\int_{\mathbb{R}} \int_{\mathbb{R}} f(t) e^{i t \lambda} d E(\lambda) d t=\int_{\mathbb{R}} \int_{\mathbb{R}} f(t) e^{i t \lambda} d t d E(\lambda)=\int_{\mathbb{R}} \widehat{f}(\lambda) d E(\lambda)=\widehat{f}(H)
$$

vanishes. For $v, w \in V$, we obtain with (ii) that

$$
\begin{aligned}
\left\langle j(v), U_{f} j(w)\right\rangle & =\int_{\mathbb{R}} f(t)\left\langle j(v), U_{t} j(w)\right\rangle d t=\int_{\mathbb{R}} f(t) \int_{0}^{\infty} e^{i t \lambda} d \mu^{v, w}(\lambda) d t \\
& =\int_{0}^{\infty} \int_{\mathbb{R}} f(t) e^{i t \lambda} d t d \mu^{v, w}(\lambda)=\int_{0}^{\infty} \widehat{f}(\lambda) d \mu^{v, w}(\lambda)=0
\end{aligned}
$$

if $\widehat{f}$ vanishes on $\mathbb{R}_{+}$. This proves that $j(V) \subseteq \operatorname{ker}\left(U_{f}\right)$ and since $U_{f}$ is an intertwining operator and the subspace $j(V) \subseteq \mathcal{H}_{\psi}$ is generating, it follows that $U_{f}=0$. This implies that $H \geq 0$.

(iii) $\Rightarrow$ (i): Write $U_{t}:=U_{t}^{\psi}=e^{i t H}$ and assume that $H \geq 0$. The spectral calculus for selfadjoint operators now implies that $\widehat{U}_{z}:=e^{i z H}, \operatorname{Im} z \geq 0$ defines a strongly continuous representation on the 
upper half plane $\mathbb{C}_{+}$which is holomorphic on the interior and whose range consists of contractions $($Ne00, Ch. VI]). Then

$$
\widehat{\psi}(z)(v, w)=\left\langle j(v), \widehat{U}_{z} j(w)\right\rangle=\left\langle j(v), e^{i z H} j(w)\right\rangle, \quad v, w \in V, \operatorname{Im} z \geq 0
$$

provides the bounded analytic extension of $\psi$ to $\mathbb{C}_{+}$.

Now we assume that (i)-(iii) are satisfied. Writing $\psi(t)(v, w)=\left\langle j(v), U_{t} j(w)\right\rangle$ for a linear map $j: V \rightarrow \mathcal{H}$ and a unitary one-parameter group $U_{t}=e^{i t H}$ on $\mathcal{H}$, we have $H \geq 0$ by (iii) and

$$
f\left(t, \tau^{\varepsilon}\right)=\left\langle j(v), e^{-|t| H} j(w)\right\rangle,
$$

so that the positive definiteness of $f$ follows from the positive definiteness of the function $t \mapsto e^{-|t| H}$ on $\mathbb{R}([$ NÓ14a, Prop. 4.1]).

\section{A Some background on positive definite kernels}

In this appendix we collect precise statements of some basic facts on positive definite kernels and functions to keep the paper more self-contained.

\section{A.1 Form-valued positive definite kernels}

Definition A.1. Let $X$ be a set and $V$ be a real vector space. We write $\operatorname{Bil}(V)=\operatorname{Bil}(V, \mathbb{C})$ for the space of complex-valued bilinear forms on $V$. We call a map $K: X \times X \rightarrow \operatorname{Bil}(V)$ a positive definite kernel if the associated scalar-valued kernel

$$
K^{b}:(X \times V) \times(X \times V) \rightarrow \mathbb{C}, \quad K^{b}((x, v),(y, w)):=K(x, y)(v, w)
$$

is positive definite 3

The corresponding reproducing kernel Hilbert space $\mathcal{H}_{K^{b}} \subseteq \mathbb{C}^{X \times V}$ is generated by the elements $K_{x, v}^{b}$, $x \in X, v \in V$, with the inner product

$$
\left\langle K_{(x, v)}^{\mathrm{b}}, K_{(y, w)}^{\mathrm{b}}\right\rangle=K(x, y)(v, w)=: K^{\mathrm{b}}((x, v),(y, w))=: K_{y, w}^{\mathrm{b}}(x, v),
$$

so that, for all $f \in \mathcal{H}_{K^{\mathrm{b}}}$, we have

$$
f(x, v)=\left\langle K_{x, v}^{b}, f\right\rangle .
$$

We identify $\mathcal{H}_{K^{b}}$ with a subspace of $\left(V^{*}\right)^{X}$ by identifying $f \in \mathcal{H}_{K^{b}}$ with the function $f^{*}: X \rightarrow V^{*}, f^{*}(x):=$ $f(x, \cdot)$. We call

$$
\mathcal{H}_{K}:=\left\{f^{*}: f \in \mathcal{H}_{K^{b}}\right\} \subseteq\left(V^{*}\right)^{X}
$$

the (vector-valued) reproducing kernel space associated to $K$. The elements

$$
K_{x, v}:=\left(K_{x, v}^{b}\right)^{*} \quad \text { with } \quad K_{x, v}(y)=K(y, x)(\cdot, v) \quad \text { for } \quad x, y \in X, v, w \in V
$$

then form a dense subspace of $\mathcal{H}_{K}$ with

$$
\left\langle K_{x, v}, K_{y, w}\right\rangle=K(x, y)(v, w) .
$$

\footnotetext{
${ }^{3}$ This definition is adapted to our convention that scalar products are linear in the second argument. Accordingly, a kernel $K: X \times X \rightarrow \operatorname{Bil}(V)$ is positive definite in the sense of Definition A.1 if and only if the kernel $(x, y) \mapsto K(x, y)^{\top}$ is positive definite in the sense of [NÓ15].
} 
Example A.2. If $V$ is a complex Hilbert space, $X$ is a set and $K: X \times X \rightarrow B(V)$ is an operator-valued kernel, then $K$ is called positive definite if the corresponding kernel

$$
\widetilde{K}:(X \times V) \times(X \times V) \rightarrow \mathbb{C}, \quad \widetilde{K}((x, v),(y, w)):=\langle v, K(x, y) w\rangle
$$

is positive definite ([Ne0, Def. I.1.1]), and this means that the kernel

$$
K^{\prime}: X \times X \rightarrow \operatorname{Sesq}(V) \subseteq \operatorname{Bil}(V), \quad K^{\prime}(x, y)(v, w):=\langle v, K(x, y) w\rangle
$$

is positive definite.

If $X=G$ is a group and the kernel $K$ is invariant under right translations, then it is of the form $K(g, h)=\varphi\left(g h^{-1}\right)$ for a function $\varphi: G \rightarrow \operatorname{Bil}(V)$.

Definition A.3. Let $G$ be a group and let $V$ be a real vector space. A function $\varphi: G \rightarrow \operatorname{Bil}(V)$ is said to be positive definite if the $\operatorname{Bil}(V)$-valued kernel $K(g, h):=\varphi\left(g h^{-1}\right)$ is positive definite.

The following proposition ([NÓ15, Prop. A.4]) generalizes the GNS construction to form-valued positive definite functions on groups.

Proposition A.4. (GNS-construction) Let $V$ be a real vector space.

(a) Let $\varphi: G \rightarrow \operatorname{Bil}(V)$ be a positive definite function. Then $\left(U_{g}^{\varphi} f\right)(h):=f(h g)$ defines a unitary representation of $G$ on the reproducing kernel Hilbert space $\mathcal{H}_{\varphi} \subseteq\left(V^{*}\right)^{G}$ with kernel $K(g, h)=\varphi\left(g h^{-1}\right)$ and the range of the map

$$
j: V \rightarrow \mathcal{H}_{\varphi}, \quad j(v)(g)(w):=\varphi(g)(w, v), \quad j(v)=K_{1, v}^{b},
$$

is a cyclic subspace, i.e., $U_{G}^{\varphi} j(V)$ spans a dense subspace of $\mathcal{H}$. We then have

$$
\varphi(g)(v, w)=\left\langle j(v), U_{g}^{\varphi} j(w)\right\rangle \quad \text { for } \quad g \in G, v, w, \in V .
$$

(b) If, conversely, $(U, \mathcal{H})$ is a unitary representation of $G$ and $j: V \rightarrow \mathcal{H}$ a linear map whose range is cyclic, then

$$
\varphi: G \rightarrow \operatorname{Bil}(V), \quad \varphi(g)(v, w):=\left\langle j(v), U_{g} j(w)\right\rangle
$$

is a $\operatorname{Bil}(V)$-valued positive definite function and $(U, \mathcal{H})$ is unitarily equivalent to $\left(U^{\varphi}, \mathcal{H}_{\varphi}\right)$.

Remark A.5. If $\varphi: G \rightarrow \operatorname{Bil}(V)$ is a positive definite function, then (32) shows that,if $\widetilde{V}:=\overline{j(V)}$, which is the real Hilbert space defined by completing $V$ with respect to the positive semidefinite form $\varphi(\mathbf{1})$, then

$$
\widetilde{\varphi}(g)(v, w)=\left\langle v, U_{g} w\right\rangle
$$

defines a positive definite function

$$
\widetilde{\varphi}: G \rightarrow \operatorname{Bil}(\widetilde{V}) \quad \text { with } \quad \widetilde{\varphi}(g)(j(v), j(w))=\varphi(g)(v, w) \quad \text { for } \quad v, w \in V .
$$

Therefore it often suffices to consider $\operatorname{Bil}(V)$-valued positive definite functions for real Hilbert space $V$ for which $\varphi(\mathbf{1})$ is a positive definite hermitian form on $V$ whose real part is the scalar product on $V$. In terms of (32), this means that $j: V \rightarrow \mathcal{H}$ is an isometric embedding of the real Hilbert space $V$. 


\section{A.2 Products of operator-valued kernels}

Lemma A.6. If $K_{j}: X \times X \rightarrow B(V), j=1,2$, are two positive definite kernels with the property that

$$
K_{1}(x, y) K_{2}\left(x^{\prime}, y^{\prime}\right)=K_{2}\left(x^{\prime}, y^{\prime}\right) K_{1}(x, y) \quad \text { for } \quad x, x^{\prime}, y, y^{\prime} \in X,
$$

then the product kernel $K:=K_{1} \cdot K_{2}$ is also positive definite.

Proof. Let $x_{1}, \ldots, x_{k}$. We have to show that the operator

$$
C:=\left(K_{1}\left(x_{j}, x_{k}\right) K_{2}\left(x_{j}, x_{k}\right)\right)_{1 \leq j, k \leq n} \in M_{n}(B(V)) \cong B\left(V^{n}\right)
$$

is positive (cf. $\mathbb{N e 0 0}$, Rem. I.1.3]).

Let $\mathcal{A}_{j} \subseteq B(V)$ denote the von-Neumann algebra generated by the values of $K_{j}$. Then $\mathcal{A}_{1}$ and $\mathcal{A}_{2}$ commute. Further, the matrices

$$
A^{(\ell)}:=\left(K_{\ell}\left(x_{j}, x_{k}\right)\right)_{1 \leq j, k \leq n} \in M_{n}\left(\mathcal{A}_{\ell}\right), \quad \ell=1,2,
$$

are positive, so that La95, Lemma 4.3] implies that the matrix

$$
D:=\left(K_{1}\left(x_{j}, x_{k}\right) \otimes K_{2}\left(x_{j}, x_{k}\right)\right) \in M_{n}\left(\mathcal{A}_{1} \otimes \mathcal{A}_{2}\right)
$$

is positive. Since $C$ is the image of $D$ under the canonical representation of $M_{n}\left(\mathcal{A}_{1} \otimes \mathcal{A}_{2}\right)$ on $V^{n}$, it follows that $C$ is positive.

\section{A.3 From real to complex-valued kernels}

In this section we take a brief look at the interplay between real and complex-valued positive definite kernels. Here Corollary A.9 is of central importance because it shows how the positive definiteness of a complex-valued form $h=\gamma+i \omega$ on a real vector space $V$ leads to a skew-symmetric contraction on the real Hilbert space $V_{\gamma}$.

Lemma A.7. Let $K: X \times X \rightarrow \mathbb{C}$ be a positive definite kernel. Then the corresponding Hilbert space $\mathcal{H}_{K} \subseteq \mathbb{C}^{X}$ is invariant under complex conjugation such that $\sigma(f):=\bar{f}$ defines an antilinear isometry on $\mathcal{H}_{K}$ if and only if $K$ is real-valued.

Proof. The invariance requirement implies the relation

$$
\left\langle f, K_{x}\right\rangle=\overline{f(x)}=\left\langle K_{x}, \sigma(f)\right\rangle=\left\langle f, \sigma\left(K_{x}\right)\right\rangle \quad \text { for } \quad f \in \mathcal{H}_{K},
$$

and therefore $\sigma\left(K_{x}\right)=K_{x}$, i.e., $K$ is real-valued. If, conversely, $K$ is real-valued, then $\mathcal{H}_{K}=\mathcal{H}_{K}^{\mathbb{R}} \oplus i \mathcal{H}_{K}^{\mathbb{R}}$ is an orthogonal sum of real Hilbert spaces, so that complex conjugation acts on $\mathcal{H}_{K}$ as an isometry.

Proposition A.8. Let $A, B: X \times X \rightarrow \mathbb{R}$ be real kernels on the set $X$. Then the kernel

$$
K=A+i B: X \times X \rightarrow \mathbb{C}
$$

is positive definite if and only if:

(a) A is positive definite, and 
(b) there exists a skew-symmetric contractive operator $C$ on the real reproducing kernel Hilbert space $\mathcal{H}_{A}^{\mathbb{R}} \subseteq \mathbb{R}^{X}$ with

$$
B(x, y)=\left\langle A_{x}, C A_{y}\right\rangle=\left(C A_{y}\right)(x) \quad \text { for } \quad x, y \in X .
$$

Proof. Necessity: If $K$ is positive definite, then so is $\bar{K}=A-i B$, and this implies that $A=\frac{1}{2}(K+\bar{K})$ is positive definite. As $A-i B=2 A-K$ is positive definite, [Ne00, Thm. I.2.8] 4 implies the existence of a bounded operator $D \geq 0$ on the complex reproducing kernel Hilbert space $\mathcal{H}_{A} \subseteq \mathbb{C}^{X}$ with

$$
K_{y}(x)=K(x, y)=\left\langle A_{x}, D A_{y}\right\rangle=\left(D A_{y}\right)(x) \quad \text { for } \quad x, y \in X .
$$

¿From Lemma A.7 we know that $\mathcal{H}_{A}=\mathcal{H}_{A}^{\mathbb{R}} \oplus i \mathcal{H}_{A}^{\mathbb{R}}$. ¿From the relation $A_{y}+i B_{y}=D A_{y}$ for every $y \in X$ and the fact that $B$ is real-valued it thus follows that $D=\mathbf{1}+i C$ for a bounded operator $C$ on $\mathcal{H}_{A}^{\mathbb{R}}$ satisfying $C A_{y}=B_{y}$ for every $y \in X$. Now $D=D^{*} \geq 0$ implies that $C=-C^{\top}$ is a contraction and

$$
B(x, y)=\left(C A_{y}\right)(x)=\left\langle A_{x}, C A_{y}\right\rangle \quad \text { for } \quad x, y \in X .
$$

Sufficiency: Suppose, conversely, that $A$ is positive definite and that $C$ is a skew-symmetric contraction on the real Hilbert space $\mathcal{H}_{A}^{\mathbb{R}}$. Then the hermitian operator $\mathbf{1}+i C$ on $\mathcal{H}_{A}^{\mathbb{C}}$ is non-negative, and therefore its symbol

$$
K(x, y):=\left((\mathbf{1}+i C) A_{y}\right)(x)=A(x, y)+i\left(C A_{y}\right)(x)
$$

is a positive definite kernel on $X$.

Corollary A.9. Let $V$ be a real vector space, let $\gamma: V \times V \rightarrow \mathbb{R}$ be a symmetric, let $\omega: V \times V \rightarrow \mathbb{R}$ be a skew-symmetric bilinear form and consider the corresponding hermitian form $h:=\gamma+i \omega$. Then the following are equivalent:

(i) $h$ is a positive definite kernel on $V$.

(ii) $\gamma$ is positive semidefinite and there exists a skew-symmetric bounded operator $C$ on the real Hilbert space $V_{\gamma}$ obtained by completing $V /\{v \in V: \gamma(v, v)=0\}$ such that $\omega(v, w)=\langle[v], C[w]\rangle_{V_{\gamma}}$, where $[v]$ denotes the image of $v$ in $V_{\gamma}$.

(iii) $\gamma$ is positive semidefinite and

$$
\omega(v, w)^{2} \leq \gamma(v, v) \gamma(w, w) \quad \text { for } \quad v, w \in V .
$$

Proof. (i) $\Leftrightarrow$ (ii): In view of Proposition A.8, the kernel $h$ is positive definite if and only if the kernel $\gamma$ is positive definite, i.e., $\gamma$ is a positive semidefinite form, and the kernel $\omega$ can be written as

$$
\omega(v, w)=\langle[v], C[w]\rangle_{V_{\gamma}} \quad \text { for } \quad v, w \in V,
$$

where $C$ is a skew-symmetric contraction on the real Hilbert space $V_{\gamma}$.

(ii) $\Rightarrow$ (iii): (34) and $\|C\| \leq 1$ imply that

$$
\omega(v, w)^{2} \leq\|C\|^{2}\|[v]\|^{2}\|[w]\|^{2}=\gamma(w, w) \gamma(v, v) .
$$

(iii) $\Rightarrow$ (ii): Suppose, conversely, that $\gamma$ is positive semidefinite and that (33) is satisfied. Then $\omega$ defines a continuous bilinear form on the real Hilbert space $V_{\gamma}$ with norm $\leq 1$. Hence there exists a skew-symmetric contraction $C \in B\left(V_{\gamma}\right)$ satisfying (34). This proves the corollary.

\footnotetext{
${ }^{4}$ For two positive definite kernels $K$ and $Q$ on a set $X$, the relation $\mathcal{H}_{K} \subseteq \mathcal{H}_{Q}$ is equivalent to $\lambda Q-K$ being positive definite for some $\lambda>0$, and this in turn is equivalent to the existence of a bounded positive operator $B$ on $\mathcal{H}_{Q}$ with $\|B\| \leq \lambda$ satisfying $K(x, y)=\left\langle Q_{x}, B Q_{y}\right\rangle=\left(B Q_{y}\right)(x)$ for $x, y \in X$ ([Ne00, Thm. I.2.8]).
} 
Lemma A.10. Let $h=\gamma+i \omega$ be a positive definite kernel as in Corollary A.9, let $\mathcal{H}_{h} \subseteq \operatorname{Hom}(V, \mathbb{C})$ be the corresponding reproducing kernel Hilbert space and let $j: V \rightarrow \mathcal{H}_{h}, j(v)=h(\cdot, v)$ the canonical map. Then the following assertions hold:

(i) $j$ is injective if and only if $\gamma$ is positive definite, i.e., defines an inner product on $V$.

(ii) The complex linear extension $j_{\mathbb{C}}: V_{\mathbb{C}} \rightarrow \mathcal{H}_{h}, v+i w \mapsto j(v)+i \cdot j(w)$ is injective if and only if

$$
\omega(v, w)^{2}<\gamma(v, v) \gamma(w, w) \quad \text { for } \quad 0 \neq v, w \in V .
$$

(iii) Suppose that $\gamma$ is positive definite, that $(V, \gamma)$ is complete and that $\omega(v, w)=\langle[v], C[w]\rangle$ for an operator $C$ on $\mathcal{H}_{\gamma}^{\mathbb{R}} \cong(V, \gamma)$. Then $j_{\mathbb{C}}$ is injective if and only if $\|C v\|<\|v\|$ for every non-zero $v \in \mathcal{H}_{\gamma}^{\mathbb{R}}$.

Proof. (i) In view of $\langle j(v), j(w)\rangle=\langle h(\cdot, v), h(\cdot, w)\rangle=h(v, w)$, we have $\|j(v)\|^{2}=h(v, v)=\gamma(v, v)$, so that $j$ is injective if and only if $\gamma$ is positive definite.

(ii) First we calculate

$$
\begin{aligned}
\left\|j_{\mathbb{C}}(v+i w)\right\|^{2} & =\|j(v)+i \cdot j(w)\|^{2}=\gamma(v, v)+\gamma(w, w)+2 \operatorname{Re}\langle j(v), i \cdot j(w)\rangle \\
& =\gamma(v, v)+\gamma(w, w)+2 \operatorname{Re} i h(w, v)=\gamma(v, v)+\gamma(w, w)+2 \omega(v, w) .
\end{aligned}
$$

Writing $\omega(v, w)=\left\langle\gamma_{w}, C \gamma_{v}\right\rangle$ as in (34), it follows that $j_{\mathbb{C}}(v+i w)=0$ is equivalent to

$$
2\left\langle\gamma_{v}, C \gamma_{w}\right\rangle=\left\langle\gamma_{v}, \gamma_{v}\right\rangle+\left\langle\gamma_{w}, \gamma_{w}\right\rangle
$$

Next we observe that $j(v)=-i \cdot j(w)$ implies $\gamma(v, v)=\|j(v)\|^{2}=\|j(w)\|^{2}=\gamma(w, w)$, which leads to

$$
\left\langle\gamma_{v}, C \gamma_{w}\right\rangle=\left\|\gamma_{v}\right\|^{2}=\left\|\gamma_{w}\right\|^{2}=\left\|\gamma_{v}\right\| \cdot\left\|\gamma_{w}\right\| .
$$

As $C$ is a contraction, this is equivalent to $C \gamma_{v}=\gamma_{w}$ by the Cauchy-Schwarz inequality.

If, conversely, there exists a non-zero $v \in V$ with $C \gamma_{v}=\gamma_{w}$ and $\gamma(v, v)=\gamma(w, w)$, then $j_{\mathbb{C}}(v+i w)=0$ by (35). This proves (ii).

(iii) If $(V, \gamma)$ is complete, $j(V) \cong(V, \gamma)$ is closed in $\mathcal{H}_{h}$. Therefore $C j(V) \subseteq j(V)$, and (iii) follows from the preceding discussion.

Remark A.11. If $V \subseteq \mathcal{H}$ is a standard real subspace (Definition 2.4), then the kernel $h(v, w):=\langle v, w\rangle$ on $V$ has the property that the corresponding reproducing kernel Hilbert space is $\mathcal{H}$ and the inclusion is the corresponding map $j: V \rightarrow \mathcal{H}$. In particular, its complex linear extension is injective.

If, conversely, $h=\gamma+i \omega$ is a positive definite bilinear kernel on a real vector space $V$, then $j(V)$ is a standard real subspace of the corresponding complex Hilbert space $\mathcal{H}_{h}$ if and only if $(V, \gamma)$ is complete (which is equivalent to the closedness of $j(V)$ ) and the complex linear extension $j_{\mathbb{C}}: V_{\mathbb{C}} \rightarrow \mathcal{H}_{h}$ is injective, which is equivalent to $j(V) \cap i \cdot j(V)=\{0\}$ (cf. Lemma A.10(iii)).

Example A.12. Consider the context of Proposition A.8 where $K=A+i B$ is a positive definite kernel and $C \in B\left(\mathcal{H}_{A}^{\mathbb{R}}\right)$ is such that $B_{y}=C A_{y}$ for $y \in X$. Then

$$
V:=(\mathbf{1}+i C) \mathcal{H}_{A}^{\mathbb{R}} \subseteq \mathcal{H}_{A}
$$

is a real subspace. For the isometric antilinear involution defined on $\mathcal{H}_{A}$ by $\sigma(f)=\bar{f}$, we then have for every $f \in \mathcal{H}_{A}^{\mathbb{R}}$ the relation

$$
\langle\sigma(\mathbf{1}+i C) f,(\mathbf{1}+i C) f\rangle=\|f\|^{2}-\|C f\|^{2} \geq 0 .
$$

Therefore $\left(\mathcal{H}_{A}, V, \sigma\right)$ is a reflection positive real Hilbert space (Proposition B.3). 


\section{A.4 Real parts of positive definite functions}

Let $\varphi: G \rightarrow \mathbb{C}$ be a positive definite function on the group $G$. Then $\bar{\varphi}$ is also positive definite, so that $\operatorname{Re} \varphi=\frac{1}{2}(\varphi+\bar{\varphi})$ is positive definite as well. ¿From Lemma A.7(a) we know that a positive definite function $\varphi$ on $G$ is real-valued if and only if the corresponding reproducing kernel Hilbert space $\mathcal{H}_{\varphi}$ is invariant under conjugation with $\|\bar{f}\|=\|f\|$ for $f \in \mathcal{H}_{\varphi}$. Based on these observations, one would like to understand the set of all positive definite functions with a given real part. A natural description of this set in the spirit of the present paper is provided by the following theorem.

Theorem A.13. (Complex extensions of real positive definite functions) Let $\varphi: G \rightarrow \mathbb{R}$ be a positive definite function and let $\left(U^{\varphi}, \mathcal{H}_{\varphi}^{\mathbb{R}}\right)$ denote the corresponding orthogonal representation on the real reproducing kernel space $\mathcal{H}_{\varphi}^{\mathbb{R}} \subseteq \mathbb{R}^{G}$ by right translations: $\left(U^{\varphi}(g) f\right)(h):=f(h g)$. Then the following assertions hold:

(a) For each skew-symmetric contraction $C$ on $\mathcal{H}_{\varphi}$ commuting with $U^{\varphi}(G)$, the function $\varphi_{C}:=\varphi+i C \varphi \in$ $\mathcal{H}_{\varphi} \subseteq \mathbb{C}^{G}$ is positive definite. Here we consider $\varphi$ as an element of the real Hilbert space $\mathcal{H}_{\varphi}^{\mathbb{R}} \subseteq \mathbb{R}^{G}$.

(b) Each positive definite function $\widehat{\varphi}$ with $\operatorname{Re} \widehat{\varphi}=\varphi$ is of the form $\varphi_{C}$ for a unique skew-symmetric contraction $C$ on $\mathcal{H}_{\varphi}$ commuting with $U^{\varphi}(G)$.

Proof. (a) Clearly $\mathcal{H}_{\varphi}=\mathcal{H}_{\varphi}^{\mathbb{R}} \oplus i \mathcal{H}_{\varphi}^{\mathbb{R}}$ is the Hilbert space complexification of $\mathcal{H}_{\varphi}^{\mathbb{R}}$ (Lemma A.7). On $\mathcal{H}_{\varphi}$ the operator $B:=\mathbf{1}+i C$ is positive because it is hermitian and $\|C\| \leq 1$. Let $K(x, y):=\varphi\left(x y^{-1}\right)$ be the kernel corresponding to $\varphi$ which satisfies $K_{y}=U^{\varphi}(y)^{-1} \varphi$. Then the associated kernel

$$
\begin{aligned}
K^{B}(x, y) & :=\left\langle B K_{y}, K_{x}\right\rangle=\left\langle B U^{\varphi}(y)^{-1} \varphi, U^{\varphi}(x)^{-1} \varphi\right\rangle=\left\langle U^{\varphi}(y)^{-1} B \varphi, U^{\varphi}(x)^{-1} \varphi\right\rangle \\
& =\left\langle U^{\varphi}\left(x y^{-1}\right)(\mathbf{1}+i C) \varphi, \varphi\right\rangle=((\mathbf{1}+i C) \varphi)\left(x y^{-1}\right)
\end{aligned}
$$

is positive definite (cf. [Ne0, Lemma I.2.4]), and this means that $\varphi+i C \varphi$ is a positive definite function.

(b) If $\widehat{\varphi}=\varphi+i \psi$ is positive definite with $\varphi, \psi$ real-valued, then write $K=A+i B$ for the corresponding kernels:

$$
K(x, y)=\widehat{\varphi}\left(x y^{-1}\right), \quad A(x, y)=\varphi\left(x y^{-1}\right) \quad \text { and } \quad B(x, y)=\psi\left(x y^{-1}\right) .
$$

Then Proposition A.8 implies that $\varphi$ is positive definite and that there exists a skew-symmetric contraction $C \in B\left(\mathcal{H}_{\varphi}^{\mathbb{R}}\right)$ with

$$
\psi\left(x y^{-1}\right)=\left(C A_{y}\right)(x)=\left\langle C U^{\varphi}(y)^{-1} \varphi, U^{\varphi}(x)^{-1} \varphi\right\rangle .
$$

Since this kernel on $G \times G$ is invariant under right translations and $U^{\varphi}(G) \varphi$ is total in $\mathcal{H}_{\varphi}^{\mathbb{R}}$, it follows that $C$ commutes with $U^{\varphi}(G)$. This in turn leads to

$$
\psi\left(x y^{-1}\right)=\left\langle C \varphi, U^{\varphi}\left(y x^{-1}\right) \varphi\right\rangle=(C \varphi)\left(x y^{-1}\right)
$$

and hence to $\psi=C \varphi$.

\section{B Standard real subspaces via contractions}

In this section we show how standard real subspaces can be parametrized in a very convenient way by skew-symmetric contractions in real Hilbert spaces. The survey article [Lo08] is an excellent source for the theory of standard real subspace. 


\section{B.1 Skew symmetric contractions}

Lemma B.1. Let $C$ be a skew-symmetric contraction on the real Hilbert space $E$ and $V:=(\mathbf{1}+i C) E \subseteq E_{\mathbb{C}}$. For $0 \neq v \in E$, the following are equivalent:

(i) $C^{2} v=-v$.

(ii) $\|C v\|=\|v\|$.

(iii) There exists $0 \neq w \in V$ with $\langle C v, w\rangle=\|v\|\|w\|$.

(iv) $(\mathbf{1}+i C) v \in V \cap i V$.

Proof. (i) $\Leftrightarrow$ (ii): First we observe that $\|v\|^{2}-\|C v\|^{2}=\left\langle\left(\mathbf{1}+C^{2}\right) v, v\right\rangle$. In view of the positivity of $\mathbf{1}+C^{2}$, the relation $\left\langle\left(\mathbf{1}+C^{2}\right) v, v\right\rangle=0$ is equivalent to $\left(\mathbf{1}+C^{2}\right) v=0$.

(ii) $\Leftrightarrow$ (iii) follows from $\max \{\langle C v, w\rangle: w \in E,\|w\| \leq 1\}=\|C v\| \leq\|v\|$.

(iv) $\Leftrightarrow$ (i): For $w \in E$, the condition $(\mathbf{1}+i C) v=i(\mathbf{1}+i C) w$ is equivalent to $C w=-v$ and $w=C v$. Such an element $w$ exists if and only if $C^{2} v=-v$.

Lemma B.2. For a skew-symmetric contraction $C$ on the real Hilbert space $E$ and $V:=(\mathbf{1}+i C) E \subseteq E_{\mathbb{C}}$, the following are equivalent:

(i) $C^{2}+\mathbf{1}$ is injective.

(ii) $\|C v\|<\|v\|$ for every non-zero $v \in E$.

(iii) $\langle C v, w\rangle<\|v\|\|w\|$ for non-zero elements $v, w \in E$.

(iv) $V \cap i V=\{0\}$.

(v) The operators $\mathbf{1} \pm i C$ on $E_{\mathbb{C}}$ are injective.

(vi) $V+i V$ is dense in $E_{\mathbb{C}}$.

(vii) $V$ is a standard real subspace.

Proof. The equivalence of (i)-(iv) follows immediately from Lemma B.1.

Further, (iv) can also be formulated as: $(\mathbf{1}+i C)(v+i w)=0$ for $v, w \in E$ implies $v+i w=0$, which in turn means that $\mathbf{1}+i C$ is injective. This in turn is equivalent to $\mathbf{1}-i C$ being injective. Therefore (iv) is equivalent to $(\mathrm{v})$.

As $V+i V=(\mathbf{1}+i C) E_{\mathbb{C}}=\operatorname{im}(\mathbf{1}+i C)$, this complex subspace is dense if and only if the hermitian operator $\mathbf{1}+i C$ has dense range, and this is equivalent to $\mathbf{1}+i C$ being injective. Therefore (v) and (vi) are also equivalent.

Next we observe that $V$ is closed because

$$
\|(\mathbf{1}+i C) v\|^{2}=\|v\|^{2}+\|C v\|^{2} \geq\|v\|^{2} \quad \text { for } \quad v \in E
$$

shows that the range $V$ of the operator $1+i C: E \rightarrow E_{\mathbb{C}}$ is closed. Since (iv) and (vi) are equivalent, they are therefore equivalent to $V$ being a standard real subspace. 
Proposition B.3. Let $E$ be a real Hilbert space, $C$ be a skew-symmetric contraction on $E, E_{\mathbb{C}}$ be the complexification of $E$ and $\sigma: E_{\mathbb{C}} \rightarrow E_{\mathbb{C}}, a+i b \mapsto a-i b$ complex conjugation on $E_{\mathbb{C}}$. Then the real subspace

$$
V:=(1+i C) E \subseteq E_{\mathbb{C}}
$$

has the following properties:

(i) Let $E_{0}=\operatorname{ker}\left(C^{2}+\mathbf{1}\right)$ and $E_{1}=E_{0}^{\perp}$, so that $E=E_{0} \oplus E_{1}$. Then $C_{0}:=\left.C\right|_{E_{0}}$ is a complex structure on $E_{0}$ and $V_{0}:=(\mathbf{1}+i C) E_{0} \subseteq E_{\mathbb{C}}$ is the $(-i)$-eigenspace of $C$. It coincides with $V \cap i V$. In particular it is a complex subspace of $E_{\mathbb{C}}$. The subspace $V_{1}:=(1+i C) E_{1}$ is a standard real subspace of $E_{1, \mathbb{C}}$.

(ii) If $V=V_{1}$, then the corresponding modular objects are given by $(\Delta, J)=\left(\left(\frac{1-i C}{1+i C}\right)^{2}, \sigma\right)$.

Proof. (i) For $a, b \in E$, the relation $C(a+i b)=-i(a+i b)$ is equivalent to $C a=b$ and $C b=-a$, i.e., to $a+i b \in V_{0}$. Therefore $V_{0}$ is the $(-i)$-eigenspace of $C$ in $E_{\mathbb{C}}$. ¿From Lemma B.1(iv) we further obtain $V \cap i V=V_{0}$. For $V_{1}:=(\mathbf{1}+i C) E_{1}$, we thus have $V_{1} \cap i V_{1}=\{0\}$, so that Lemmas B.2(vii) implies that $V_{1}$ is a standard real subspace of $E_{1, \mathbb{C}}$.

(ii) If $V=V_{1}$, then

$$
\Delta:=\left(\frac{\mathbf{1}-i C}{\mathbf{1}+i C}\right)^{2}
$$

is a positive selfadjoint operator on $E_{\mathbb{C}}$ with domain $(\mathbf{1}+i C)^{2} E_{\mathbb{C}}$. Further $\Delta^{1 / 2}=(\mathbf{1}-i C)(\mathbf{1}+i C)^{-1}$ has domain $V_{\mathbb{C}}$

Since $\sigma \Delta \sigma=\Delta^{-1}$ by (36), $S:=\sigma \Delta^{1 / 2}$ is an unbounded antilinear involution with

$$
\operatorname{Fix}(S)=\left\{\xi \in \mathcal{D}\left(\Delta^{1 / 2}\right)=V_{\mathbb{C}}: S \xi=\xi\right\} .
$$

For $\xi=(\mathbf{1}+i C) v, v \in E_{\mathbb{C}}$, we have

$$
S \xi=\sigma \Delta^{1 / 2} \xi=\sigma(\mathbf{1}-i C) v=(\mathbf{1}+i C) \sigma(v),
$$

so that $S \xi=\xi$ is equivalent to $v \in V$. We conclude that $\operatorname{Fix}(S)=V$. This proves (ii).

Remark B.4. Let $C$ be a skew-symmetric contraction on the real Hilbert space $E$. Then the selfadjoint operator $C^{2}+\mathbf{1}$ is invertible if and only if $-\mathbf{1} \notin \operatorname{Spec}\left(C^{2}\right)$, which is equivalent to $\mathbf{1} \notin \operatorname{Spec}(i C)$, where $i C$ is considered as a selfadjoint operator on the complex Hilbert space $E_{\mathbb{C}}$. This, in turn, is equivalent to the invertibility of $\mathbf{1}+i C$ and hence to the boundedness of $(\mathbf{1}-i C)(\mathbf{1}+i C)^{-1}$.

\section{B.2 Real reflection positivity and standard subspaces}

In this section we relate standard real subspaces to reflection positive real Hilbert spaces of the form $\left(E_{\mathbb{C}}, V, \sigma\right)$, where $\sigma$ is the complex conjugation on the complexification $E_{\mathbb{C}}$ of a real Hilbert space. This sheds an interesting light on the close connection between standard real subspaces and reflection positivity.

Lemma B.5. Let $E$ be a real Hilbert space and $E_{\mathbb{C}}$ be its complexification. On $E_{\mathbb{C}}$ we consider the antilinear isometry defined by $\sigma(a+i b):=a-i b$. A real subspace $V \subseteq E_{\mathbb{C}}$ has the property that the form $(v, w) \mapsto\langle\sigma v, w\rangle$ is real-valued and positive semidefinite on $V$ if and only if there exists a skew-symmetric contraction $C: \mathcal{D}(C) \rightarrow E$ with $V=(\mathbf{1}+i C)(\mathcal{D}(C))$. The subspace $V$ is closed if and only if $\mathcal{D}(C)$ is closed. 
Proof. First, let $C: \mathcal{D}(C) \rightarrow E$ be a skew-symmetric contraction and put $V:=(\mathbf{1}+i C) \mathcal{D}(C)$. For $v, w \in \mathcal{D}(C)$, we then have

$$
\begin{aligned}
\langle\sigma((\mathbf{1}+i C) v),(\mathbf{1}+i C) w\rangle & =\langle(\mathbf{1}-i C) v),(\mathbf{1}+i C) w\rangle=\langle v, w\rangle+\langle-i C v, w\rangle+\langle v, i C w\rangle-\langle C v, C w\rangle \\
& =\langle v, w\rangle-\langle C v, C w\rangle=\left\langle\left(\mathbf{1}+C^{2}\right) v, w\right\rangle \in \mathbb{R} .
\end{aligned}
$$

Moreover $\mathbf{1}+C^{2} \geq 0$ implies that the form is positive semidefinite.

Conversely, let $V \subseteq E_{\mathbb{C}}$ be a real subspace which is $\sigma$-positive in the sense that the form $f(v, w):=$ $\langle\sigma v, w\rangle$ is real-valued and positive semidefinite. This assumption implies that $V \cap i E=\{0\}$. Hence there exists a real linear operator $C: \mathcal{D}(C) \rightarrow E$ for which $V=(\mathbf{1}+i C) \mathcal{D}(C)$. Since

$$
\langle\sigma(v+i C v), w+i C w\rangle=\langle v-i C v, w+i C w\rangle=\langle v, w\rangle-\langle C v, C w\rangle+i(\langle C v, w\rangle+\langle C w, v\rangle)
$$

is supposed to be real-valued,

$$
\langle C v, w\rangle+\langle v, C w\rangle=0 \quad \text { for } \quad v, w \in E .
$$

This means that $C$ is skew-symmetric on $\mathcal{D}(C)$. Further, the positivity assumption implies that $\|C v\| \leq$ $\|v\|$ for $v \in E$.

The subspace $V$ is closed if and only if the graph of $C$ is closed, which is equivalent to the closedness of $\mathcal{D}(C)$ because $C$ is a contraction.

Proposition B.6. Let $E$ be a real Hilbert space, $C$ be a skew-symmetric contraction on $E$, $E_{\mathbb{C}}$ be the complexification of $E$ and $\sigma: E_{\mathbb{C}} \rightarrow E_{\mathbb{C}}, a+i b \mapsto a-i b$ complex conjugation on $E_{\mathbb{C}}$. Then the real subspace

$$
V:=(\mathbf{1}+i C) E \subseteq E_{\mathbb{C}}
$$

has the following properties:

(i) $V$ is closed and $\sigma$-positive, so that $\left(E_{\mathbb{C}}, V, \sigma\right)$ is a reflection positive real Hilbert space.

(ii) $V^{\perp}=i \sigma(V)$, i.e., the bilinear form $\gamma_{\sigma}(\xi, \eta):=\langle\sigma \xi, \eta\rangle$ on $V$ is real-valued.

(iii) The null space of the positive semidefinite form $\gamma_{\sigma}$ on $V$ coincides with the $(-i)$-eigenspace $V_{0}$ of $C$ on $E_{\mathbb{C}}$. If $V_{0}=\{0\}$, then the unbounded positive operator

$$
F:=\sqrt{\frac{1-i C}{1+i C}}: V \rightarrow E_{\mathbb{C}}
$$

satisfies $\|F \xi\|^{2}=\langle\sigma \xi, \xi\rangle$ for $\xi \in V$, so that we can identify the real Hilbert space completion $\widehat{V}$ of $V$ with respect to $\gamma_{\sigma}$ with $\overline{F(V)}$. We further have $\sigma F \sigma=F^{-1}$.

Proof. (i) The subspace $V$ is closed because

$$
\|(\mathbf{1}+i C) v\|^{2}=\|v\|^{2}+\|C v\|^{2} \geq\|v\|^{2} \quad \text { for } \quad v \in E
$$

shows that the range of the operator $1+i C: E \rightarrow V$ is closed.

For the complex conjugation $\sigma$ on $E_{\mathbb{C}}$, we have for $v, w \in E$ the relation

$$
\begin{aligned}
\gamma_{\sigma}((\mathbf{1}+i C) v,(\mathbf{1}+i C) w) & =\langle\sigma(\mathbf{1}+i C) v,(\mathbf{1}+i C) w\rangle=\langle(\mathbf{1}-i C) v,(\mathbf{1}+i C) w\rangle \\
& =\langle(\mathbf{1}+i C)(\mathbf{1}-i C) v, w\rangle=\left\langle\left(\mathbf{1}+C^{2}\right) v, w\right\rangle \in \mathbb{R}
\end{aligned}
$$


and thus

$$
\gamma_{\sigma}((\mathbf{1}+i C) v,(\mathbf{1}+i C) v)=\|v\|^{2}-\|C v\|^{2} \geq 0 .
$$

(iii) An element $a+i b \in E_{\mathbb{C}}(a, b \in E)$ is orthogonal to $V$ with respect to the real scalar product if and only if

$$
0=\operatorname{Re}\langle a+i b, v+i C v\rangle=\langle a, v\rangle+\langle b, C v\rangle=\langle a-C b, v\rangle
$$

for every $v \in E$, and this is equivalent to $C b=a$, i.e., to $a+i b=i(b-i C b) \in i \sigma(V)$.

(iv) An element $\xi:=(\mathbf{1}+i C) v \in V$ satisfies $\langle\sigma \xi, \xi\rangle=0$ if and only if $C^{2} v=-v$, which is equivalent to

$$
(\mathbf{1}-i C) \xi=(\mathbf{1}-i C)(\mathbf{1}+i C) v=\left(\mathbf{1}+C^{2}\right) v=0,
$$

i.e., to $C \xi=-i \xi$. This implies that $V_{0} \subseteq V$ is the nullspace of $\gamma_{\sigma}$.

Now we assume that $V_{0}=\{0\}$ and $V=V_{1}$. As $\mathbf{1} \pm i C$ are non-negative hermitian operators on $E_{\mathbb{C}}$, they have a non-negative square root and $(\mathbf{1}+i C)^{-1 / 2}$ is an unbounded operator whose domain is

$$
\sqrt{\mathbf{1}+i C} E_{\mathbb{C}} \supseteq \sqrt{\mathbf{1}+i C} \sqrt{\mathbf{1}+i C} E_{\mathbb{C}}=(\mathbf{1}+i C) E_{\mathbb{C}} .
$$

This leads to an unbounded symmetric operator

$$
F:=\sqrt{\frac{1-i C}{1+i C}}: V \rightarrow E_{\mathbb{C}} .
$$

For $\xi=(\mathbf{1}+i C) v, v \in E$, we have

$$
F \xi=\sqrt{(\mathbf{1}-i C)(\mathbf{1}+i C)} v=\sqrt{\mathbf{1}+C^{2}} v,
$$

so that $\|F \xi\|^{2}=\left\langle\left(\mathbf{1}+C^{2}\right) v, v\right\rangle=\langle\sigma \xi, \xi\rangle$. Therefore $F: V \rightarrow \widehat{V}:=\overline{F(V)} \subseteq E_{\mathbb{C}}$ is the canonical map of the reflection positive real Hilbert space $\left(E_{\mathbb{C}}, V, \sigma\right)$. It satisfies

$$
\sigma F \sigma=\sqrt{\frac{1+i C}{1-i C}}=F^{-1}
$$

Remark B.7. Since $U_{t}=\Delta^{-i t}$ acts on the reflection positive Hilbert space $\left(E_{\mathbb{C}}, V, \sigma\right)$ by automorphisms, it induces on the corresponding real Hilbert space $\widehat{V}$ an orthogonal representation. The natural map $\sqrt{\mathbf{1 + C ^ { 2 }}}: E \rightarrow \widehat{V}$ in Proposition $\left[\right.$ B.6 intertwines the orthogonal representations $\left.U_{t}\right|_{E}$ and $\left.U_{t}\right|_{\widehat{V}}$.

The following proposition asserts that all standard real subspace are of the form described in Proposition B.3.

Proposition B.8. Let $V \subseteq \mathcal{H}$ be a standard real subspace with modular objects $(\Delta, J)$. Then $E:=\operatorname{Fix}(J)$ is a real Hilbert space with $\mathcal{H} \cong E_{\mathbb{C}}$ and there exists a skew-symmetric strict contraction $C: E \rightarrow E$ with $V=(\mathbf{1}+i C) E$. Then $\mathcal{D}(\Delta) \cap V$ is dense in $V$.

Proof. First we observe that $V$ is $J$-positive:

$$
\langle J \xi, \xi\rangle=\langle J S \xi, \xi\rangle=\left\langle\Delta^{1 / 2} \xi, \xi\right\rangle \geq 0 .
$$

This implies the existence of a contraction $C: \mathcal{D}(C) \rightarrow E$ with

$$
V=\Gamma(C):=(\mathbf{1}+i C) \mathcal{D}(C)
$$


(Subsection 3.2). That $C$ is strict follows from Lemma B.2 ¿From the real orthogonal decomposition $\mathcal{H}=V \oplus i J(V)($ NÓ15, Lemma 4.2(iv)]) we now obtain

$$
V^{\perp}=i J(V)=i(\mathbf{1}-i C) \mathcal{D}(C)=i \Gamma(-C)=(C+i \mathbf{1}) \mathcal{D}(C),
$$

where $\perp$ refers to the real-valued scalar product $\operatorname{Re}\langle\cdot, \cdot\rangle$ on $\mathcal{H} \cong E \oplus i E$.

If $a \in E \cap \mathcal{D}(C)^{\perp}$, then $a \in V^{\perp}=i J(V)=i \Gamma(-C)$ leads to $a=C 0=0$. Therefore $\mathcal{D}(C)$ is dense in $E$. As $V$ is closed and $\mathbf{1}+i C: \mathcal{D}(C) \rightarrow V$ is a topological isomorphism, it follows that $\mathcal{D}(C)$ is closed, and thus $\mathcal{D}(C)=E$.

As $\gamma_{J}(\xi, \eta):=\langle J \xi, \eta\rangle$ is real-valued on $V$ (recall $J V=(i V)^{\perp}$ ), we obtain for $v, w \in V$ the relation

$$
\begin{aligned}
0 & =\operatorname{Im}\langle J(\mathbf{1}+i C) v,(\mathbf{1}+i C) w\rangle=\operatorname{Im}\langle(\mathbf{1}-i C) v,(\mathbf{1}+i C) w\rangle \\
& =\operatorname{Im}\left\langle\left(\mathbf{1}-i C^{\top}\right)(\mathbf{1}-i C) v, w\right\rangle=-\left\langle\left(C^{\top}+C\right) v, w\right\rangle,
\end{aligned}
$$

so that $C^{\top}=-C$ (Lemma B.5).

It remains to show that $\mathcal{D}(\Delta) \cap V$ is dense in $V$. Since $C$ is a strict contraction, the kernel of $\mathbf{1}+C^{2}$ is trivial, resp., -1 is not an eigenvalue of $C^{2}$. Let $E_{n} \subseteq E$ be the spectral subspace of $C^{2}$ for the subset $[-1+1 / n, 1]$. This subspace is $C$-invariant and the union of these subspace is dense in $E$ because -1 is not an eigenvalue. As $(\mathbf{1}+i C) E_{n} \subseteq \mathcal{D}(\Delta)$, it follows that $\mathcal{D}(\Delta) \cap V$ is dense in $V$.

\section{B.3 Contractions and modular objects}

The following lemma describes the complex-valued scalar product on a standard real subspace in terms of the corresponding modular objects $(\Delta, J)$.

Lemma B.9. Let $V \subseteq \mathcal{H}$ be a standard real subspace, $(\Delta, J)$ be the corresponding modular objects and

$$
\langle v, w\rangle_{\mathcal{H}}=\gamma(v, w)+i \omega(v, w)
$$

be the corresponding hermitian positive definite form on $V$; in particular $\langle v, w\rangle_{V}=\gamma(v, w)$. Then

$$
\gamma(v, w)=\frac{1}{2}\left(\langle v, w\rangle+\left\langle\Delta^{1 / 2} v, \Delta^{1 / 2} w\right\rangle\right) \quad \text { and } \quad \omega(v, w)=\frac{1}{2 i}\left(\langle v, w\rangle-\left\langle\Delta^{1 / 2} v, \Delta^{1 / 2} w\right\rangle\right) .
$$

In particular, we have a strict contraction $C$ on $V$ satisfying:

$$
\omega(v, w)=\gamma(v, C w) \quad \text { and } \quad C=\left.\widehat{C}\right|_{V}, \quad \widehat{C}=i \frac{\Delta-\mathbf{1}}{\Delta+1}=i \frac{\Delta^{1 / 2}-\Delta^{-1 / 2}}{\Delta^{1 / 2}+\Delta^{-1 / 2}}=i \tanh \left(\frac{\log \Delta}{2}\right) .
$$

It satisfies

$$
\langle v, w\rangle_{\mathcal{H}}=\langle v,(\mathbf{1}+i C) w\rangle_{V_{\mathbb{C}}} \quad \text { for } \quad v, w \in V_{\mathbb{C}},
$$

so that the map $\Phi:=\sqrt{\mathbf{1}+i C}: V_{\mathbb{C}} \rightarrow V_{\mathbb{C}}$ extends to an isometric inclusion $\mathcal{H} \hookrightarrow V_{\mathbb{C}}$.

Proof. As $V \subseteq \mathcal{D}\left(\Delta^{1 / 2}\right)$ and $v=S v=J \Delta^{1 / 2} v$ or $v \in V$ (Remark 2.5), we obtain

$$
\left\langle\Delta^{1 / 2} v, \Delta^{1 / 2} w\right\rangle=\langle J v, J w\rangle=\langle w, v\rangle=\overline{\langle v, w\rangle} \quad \text { for } \quad v, w \in V .
$$

This implies (37). Next we note that $B:=\frac{\Delta-1}{\Delta+1}$ is a bounded operator on $\mathcal{H}$ which can also be written as

$$
B=\frac{\Delta^{1 / 2}-\Delta^{-1 / 2}}{\Delta^{1 / 2}+\Delta^{-1 / 2}}
$$


In this form we see that $J B J=-B$. We also note that $B$ commutes with $\Delta$, hence preserves $\mathcal{D}\left(\Delta^{1 / 2}\right)$. This leads to

$$
S B=J \Delta^{1 / 2} B=-B S,
$$

and therefore to $B V=B \operatorname{Fix}(S) \subseteq i \operatorname{Fix}(S)=i V$. In particular, $\widehat{C}:=i B$ restricts to a bounded skew-symmetric operator $C: V \rightarrow V$. If $v, w$ are contained in the dense subspace $V \cap \mathcal{D}(\Delta)$ of $V$ (Proposition B.8), we obtain

$$
\begin{aligned}
\gamma(v, C w) & =\frac{1}{2}\left(\langle v, C w\rangle+\left\langle\Delta^{1 / 2} v, \Delta^{1 / 2} C w\right\rangle\right)=\frac{1}{2}\langle(\mathbf{1}+\Delta) v, C w\rangle \\
& =\frac{1}{2}\langle v,(\mathbf{1}+\Delta) \widehat{C} w\rangle=\frac{1}{2 i}\langle v,(\mathbf{1}-\Delta) w\rangle=\omega(v, w) .
\end{aligned}
$$

Since $\omega$ and $\gamma(\cdot, C \cdot)$ are continuous on $V$, they coincide on all of $V$. By Lemma B.2, the operator $C$ is a strict contraction. By (38), we have for $v, w \in V$ the relation (39), and since both sides are sesquilinear, it also holds for $v, w \in V_{\mathbb{C}}$.

\section{References}

[AFG86] de Angelis, G. F., D. de Falco, and G. di Genova, Random fields on Riemannian manifolds: a constructive approach, Commun. Math. Phys. 103 (1986), 297-303

[Ar63] Araki, H., A lattice of von Neumann algebras associated with the quantum theory of a free Bose field, J. Mathematical Phys. 4 (1963), 1343-1362

[BJM13] Barata, J. C. A., C. D. Jäkel, and J. Mu-nd, The $P(\varphi)_{2}$ model on the de Sitter space, Preprint, arXiv:math-ph:1311.2905

[BJM15] Barata, J. C. A., C. D. Jäkel, and J. Mund, An algebraic construction of interacting quantum theories, Preprint, 2015

[Bo92] Borchers, H.-J., The CPT-Theorem in two-dimensional theories of local observables, Comm. Math. Phys. 143 (1992), 315-332

[BR96] Bratteli, O., and D. W. Robinson, "Operator Algebras and Quantum Statistical Mechanics II," 2nd ed., Texts and Monographs in Physics, Springer-Verlag, 1996.

[BR02] Bratteli, O., and D. W. Robinson, "Operator Algebras and Quantum Statistical Mechanics I," 2nd ed., Texts and Monographs in Physics, Springer-Verlag, 2002

[BB94] Bros, J., and D. Buchholz, Towards a relativistic KMS-condition, Nuclear Phys. B 429:2 (1994), 291-318

[BLS11] Buchholz, D., Lechner, G., and S. J. Summers, Warped convolutions, Rieffel deformations and the construction of quantum field theories, Comm. Math. Phys. 304:1 (2011), 95-123

[CMV01] Cuniberti, G., E. De Micheli, and G. A. Viano, Reconstruction of the thermal Green functions at real times from those at imaginary times, Comm. Math. Phys. 216 (2001), 59-83 
[DG13] Dereziński, J., and C. Gérard, "Mathematics of Quantization and Quantum Fields," Cambridge Monographs on Mathematical Physics, Cambridge University Press, Cambridge, 2013

[Di04] Dimock, J., Markov quantum fields on a manifold, Rev. Math. Phys. 16:2 (2004), 243-255

[EO73] Eckmann, J.-P., and K. Osterwalder, An application of Tomita's theory of modular Hilbert algebras: duality for free Bose Fields, J. Funct. Anal. 13 (1973), 1-12

[GJ06] Gérard, C., and C. Jäkel, On the relativistic KMS condition for the $P(\varphi)_{2}$ model, arXiv:math-ph/0609088

[GJ81] Glimm, J., and A. Jaffe, "Quantum Physics-A Functional Integral Point of View," SpringerVerlag, New York, 1981

[JR07] Jaffe, A., and G. Ritter, Quantum field theory on curved backgrounds. I. The euclidean functional integral, Comm. Math. Phys. 270 (2007), 545-572

[Jo02] Jorgensen, P. E. T., Diagonalizing operators with reflection symmetry, J. Funct. Anal. 190 (2002), 93-132

[JÓl98] Jorgensen, P. E. T., and G. Ólafsson, Unitary representations of Lie groups with reflection symmetry, J. Funct. Anal. 158 (1998), 26-88

[JÓ100] Jorgensen, P. E. T., and G. Ólafsson, Unitary representations and Osterwalder-Schrader duality, in "The Mathematical Legacy of Harish-Chandra," R. S. Doran and V. S. Varadarajan, eds., Proc. Symp. in Pure Math. 68, Amer. Math. Soc., 2000

[KL81] Klein, A., and L. Landau, Periodic Gaussian Osterwalder-Schrader positive processes and the two-sided Markov property on the circle, Pac. J. Math. 94:2 (1981), 341-367

[KL83] - From the Euclidean group to the Poincaré group via Osterwalder-Schrader positivity, Comm. Math. Phys. 87 (1983), 469-484

[La95] Lance, E. C., "Hilbert $C^{*}$-modules," London Math. Soc. Lecture Notes 210, 1995

[Lo08] Longo, R., Real Hilbert subspaces, modular theory, SL(2, R) and CFT in "Von Neumann Algebras in Sibiu", 33-91, Theta Ser. Adv. Math. 10, Theta, Bucharest, 2008

[dMV16] de Micheli, E., and G. A. Viano, Holomorphic extensions associated with series expansion, arXiv:math-ph:1602.02021

[Ne00] Neeb, K. - H., "Holomorphy and Convexity in Lie Theory," Expositions in Mathematics 28, de Gruyter Verlag, Berlin, 2000

[NÓ14a] Neeb, K.-H., G. Ólafsson, Reflection positivity and conformal symmetry, J. Funct. Anal. 266 (2014), 2174-2224

[NÓ14b] -, Reflection positive one-parameter groups and dilations, Complex Analysis and Operator Theory 9:3 (2015), 653-721 
[NÓ15] Neeb, K.-H., G. Ólafsson, Reflection positivity for the circle group, arXiv:math.RT.1411.2439; in "Proceedings of the 30th International Colloquium on Group Theoretical Methods," Journal of Physics: Conference Series 597 (2015), 012004

[NÓ16] Neeb, K.-H., G. Ólafsson, Reflection positivity on spheres and hyperboloids, in preparation

[OS73] Osterwalder, K., and R. Schrader, Axioms for Euclidean Green's functions. 1, Comm. Math. Phys. 31 (1973), 83-112

[Ra00] Ramacher, P., Modular localization of elementary systems in the theory of Wigner, J. Math. Physics 41:9 (2000), 6079-6089

[RvD77] Rieffel, M. A., and A. van Daele, A bounded operator approach to Tomita-Takesaki Theory, Pac. J. Math. 69:1 (1977), 187-220

[Sch99] Schlingemann, D., ¿From euclidean field theory to quantum field theory, Rev. Math. Phys. 11 (1999), 1151-1178

[Yn94] Yngvason, J., A note on Essential Duality, Lett. Math. Phys. 31:2 (1994), 127-141 